\title{
ON THE CONDITIONS OF INTEGRABILITY OF COVARIANT DIFFERENTIAL EQUATIONS*
}

BY

\section{J. A. SCHOUTEN}

In differential geometry conditions of integrability frequently occur, but in the cases usually investigated only the first of these conditions has to be considered. In 1922t Eisenhart and Veblen gave a necessary and sufficient condition that a geometry of paths be a Riemann geometry by using a new method of treating the conditions of integrability of higher order. Recently Veblen and Thomas have generalised this method in these Transactions $\ddagger$ and succeeded in giving a very elegant treatment of linear equations of the form

$$
\nabla_{\mu} v_{\lambda_{1} \cdots \lambda_{p}}=0
$$

and of linear equations of the form

for $p=2 . \S$

$$
\nabla_{(\mu} v_{\left.\lambda_{1} \cdots \lambda_{p}\right)}=0
$$

Now the conditions of integrability have been the subject of a great number of investigations, especially by Lie, Bouquet, Mayer, Bourlet, Delassus and Riquier, and in these papers the problem is solved for very general forms of systems of differential equations. The methods used by these authors, however, are not directly applicable in the case of covariant equations as used in differential geometry, as they are too general and not in covariant form. Therefore it must be possible to establish a method in covariant form for the treatment of covariant equations much more simple than these general methods, and more convenient for use. In the first part of this paper (pp. 442-453) we deal with equations of the form

$$
\nabla_{\mu} v_{\lambda_{1} \cdots \lambda_{p}}=w_{\mu \lambda_{1} \cdots \lambda_{p}},
$$

the right side being a function of $x^{\nu}$ and $v_{\lambda_{1} \ldots \lambda_{p}}$ only, containing no derivatives of $v_{\lambda_{1}} \cdots \lambda_{p}$. The general solution of these equations, if existing,

* Presented to the Society, September 11, 1925.

†Proceedings of the National Academy of Sciences, vol. 8 (1922), pp. 19-23. $\ddagger$ Vol. 25, pp. 551-608.

$\S$ We use in this paper the notations of the author's Der Ricci Kalkül, Berlin, J. Springer, 1924; cited in the sequel as R. K. 
depends always on a finite number of arbitrary constants. The conditions of integrability and their treatment are given in covariant form. The linear case, including as a special case the first equation of Veblen and Thomas, is treated more in detail in a separate section. In the second part (pp. 45̃3-473) we deal with equations of the form

$$
P \nabla_{\mu} v_{\lambda_{1} \cdots \lambda_{p}}=w_{\mu \lambda_{1} \cdots \lambda_{p}}
$$

the right side having the properties mentioned above and $P$ being any operator linear homogeneous in the permutations of the $p+1$ suffixes $\mu \lambda_{1} \ldots \lambda_{p}$. The treatment of such equations is possible by using the author's development of arbitrary quantities of degree $p+1$ in series of indivisible quantities. By using this development and a general theorem of Lie we find the necessary and sufficient condition that the general solution depend on a finite number of arbitrary constants. This condition being satisfied, the conditions of integrability and their treatment can be given in covariant form. The linear case, including as a special case the second equation of Veblen and Thomas, is treated more in detail in a separate section.

The case in which the general solution involves arbitrary functions is not dealt with in this paper.

\section{A. ON COVARIANT EQUATIONS ADMITTING A SOLUTION FOR THE FIRST DERIVATIVES}

1. The conditions of integrability. In this chapter we consider covariant equations of the form

$$
\nabla_{\mu} v_{\lambda_{1} \cdots \lambda_{p}}=w_{\mu \lambda_{1} \cdots \lambda_{p}} ; \quad \mu, \lambda_{1}, \ldots, \lambda_{p}=a_{1}, \ldots, a_{n},
$$

$w_{\mu \lambda_{1} \cdots \lambda_{p}}$ being a quantity, depending on $x^{\nu^{\prime}}$ and $v_{\lambda_{1} \cdots \lambda_{p}}$ only, not containing derivatives of $v_{\lambda_{1}} \cdots \lambda_{p} ; \nabla_{\mu}$ is the operator of covariant differentiation, defined by the equation*

$$
\nabla_{\mu} v_{\lambda_{1} \cdots \lambda_{p}}=\frac{\partial v_{\lambda_{1} \cdots \lambda_{p}}}{\partial x^{\mu}}+\sum_{x}^{1, \ldots, p} \Gamma_{\lambda_{x_{l}} l l}^{\nu} v_{\lambda_{1} \cdots \lambda_{x-1} \nu_{\lambda_{x+1}} \cdots \lambda_{p},}
$$

the parameters $\Gamma_{\lambda \mu}^{\nu}$ being once for all given as functions of $x^{\nu}$. In (1.1) only lower suffixes appear, but all properties derived in this chapter hold also for quantities with higher and lower suffixes.

\footnotetext{
* Signs of summation are always omitted when they belong to Greek suffixes, but never when they belong to Latin ones.
} 
Temporarily we make use of suffixes $u, v, w$, taking all values from 1 to $N=n^{p}$, and write

$$
\begin{aligned}
& v_{a_{1} \ldots a_{1}}=v_{1}, \\
& v_{a_{1} \ldots a_{1} a_{2}}=v_{2}, \text { etc., }
\end{aligned}
$$

so that (1.1) is equivalent to

$$
\frac{\partial v_{u}}{\partial x^{\mu}}=W_{\mu u} \quad\left(u=1, \cdots, N ; \mu=a_{1}, \cdots, a_{n}\right)
$$

We recapitulate briefly some well known properties of these equations.* The solutions of (1.4) satisfy the equations

$$
\frac{\partial W_{\mu u}}{\partial x^{\omega}}+\sum_{v} \frac{\partial W_{\mu u}}{\partial v_{v}} W_{\omega v}=\frac{\partial W_{\omega u}}{\partial x^{\mu}}+\sum_{v} \frac{\partial W_{\omega u}}{\partial v_{v}} W_{\mu v},
$$

which do not contain any derivatives of $v_{u}$ and may therefore be put in the form

$$
F_{x}\left(v_{1}, \cdots, v_{N}\right)=0 \quad\left(x=1, \cdots, N\left(\begin{array}{l}
n \\
2
\end{array}\right)\right)
$$

If (1.6) is identically satisfied, viz. for all the values of $v_{u}$, then (1.4) is completely integrable. In this case if $W_{\mu u}$ are functions of $x^{\nu}$, $v_{u}$, regular in the vicinity of some arbitrary given values $\underset{0}{x^{\nu}},{\stackrel{0}{v_{u}}}_{\text {, there }}$ exists one and only one system of solutions $v_{u}$, regular in this region and taking the values $v_{u}$ for $x^{\nu}=x_{0}^{\nu}$. Hence if (1.4) are completely integrable, the general solution depends on a finite number of arbitrary constants.

If equations (1.6) are not identically satisfied, there will be some of them, say $N-N^{\prime}$, independent. Then, by (1.6), $N-N^{\prime}$ of the variables $v_{u}$ are functions of the other $N^{\prime}$ and of $x^{\nu}$. Without loss of generality we may assume that these variables are $v_{N^{\prime}+1}, \cdots, v_{N}$. Then (1.6) is equivalent to

$$
v_{N^{\prime}+s}=\varphi_{s}\left(v_{1}, \cdots, v_{N^{\prime}}\right) \quad\left(s=1, \cdots, N-N^{\prime}\right)
$$

Substituting the values of $v_{N^{\prime}+s}$ from (1.7) into (1.4) we get from the first $N^{\prime}$ equations $N^{\prime}$ new equations

* Bouquet, Bulletin des Sciences Mathématiques et Astronomiques, vol. 3 (1872), pp. 265-274; Mayer, the same Bulletin, vol. 11 (1876); Mathematische Annalen, vol. 5 (1872), pp. 448-470; Bourlet, Annales de l'École Normale Supérieure, ser. 3, vol. 8 (1891), supplément, pp. 1-63. 


$$
\frac{\partial v_{a}}{\partial x^{\mu}}=W_{\mu a}^{\prime} \quad\left(a=1, \cdots, N^{\prime}\right),
$$

in which $W_{\mu a}^{\prime}$ contains only $v_{1}, \cdots, v_{N^{\prime}}$. The other $N-N^{\prime}$ equations (1.4) lead to $N-N^{\prime}$ equations containing only $v_{1}, \cdots, v_{N^{\prime}}$ and no derivatives:

$$
F_{s}^{\prime}\left(v_{1}, \cdots, v_{N^{\prime}}\right)=0 \quad\left(s=1, \cdots, N-N^{\prime}\right) .
$$

The conditions of integrability of $\left(1.4^{\prime}\right)$ are obtained by substituting the values from (1.7) into (1.5). Hence the equations (1.4') are then and only then completely integrable when $\left(1.6^{\prime}\right)$ is satisfied identically.

If equations $\left(1.6^{\prime}\right)$ are not identically satisfied, there will be some of them, say $N^{\prime}-N^{\prime \prime}$, independent. Then, by $\left(1.6^{\prime}\right), N^{\prime}-N^{\prime \prime}$ of the variables can be eliminated, giving rise to the equations

$$
\begin{gathered}
\frac{\partial v_{b}}{\partial x^{\mu}}=W_{\mu b}^{\prime \prime} \quad\left(b=1, \cdots, N^{\prime \prime}\right), \\
F_{t}^{\prime \prime}\left(v_{1}, \cdots, v_{N^{\prime \prime}}\right)=0 \quad\left(t=1, \cdots, N^{\prime}-N^{\prime \prime}\right),
\end{gathered}
$$

$W_{\mu b}^{\prime \prime}$ containing only $v_{1}, \ldots, v_{N^{\prime \prime}}$. The equations $\left(1.4^{\prime \prime}, 6^{\prime \prime}\right)$ can be treated in the same way as $\left(1.4^{\prime}, 6^{\prime}\right)$. Proceeding in this way we may arrive at a completely integrable system of $N^{*}$ equations,

$$
\frac{\partial v_{c}}{\partial x^{\mu}}=W_{\mu c}^{*} \quad\left(c=1, \ldots, N^{*}\right),
$$

$W_{\mu c}^{*}$ containing only $v_{1}, \cdots, v_{N^{*}}$, and $N-N^{*}$ equations, expressing the other $N-N^{*}$ variables as functions of $v_{1}, \ldots, v_{N^{*}}$ :

$$
v_{d}=f_{d}\left(v_{1}, \cdots, v_{N^{*}}\right) \quad\left(d=N^{*}+1, \ldots, N\right) .
$$

In this case the general solution of (1.4) depends on a finite number of arbitrary constants. But it is also possible that we may arrive at a number of independent equations between $v_{1}, \ldots, v_{N}$ greater than $N$. Then (1.4) is inconsistent.

2. The covariant form of the conditions. We will now derive a covariant form of the conditions of integrability, much more convenient than the form deduced in the preceding section. To this purpose we consider once more the process leading from $(1.4,6)$ to $\left(1.4^{\prime}, 6^{\prime}\right)$. (1.4') and $\left(1.6^{\prime}\right)$ are algebraic consequences of (1.6) and the first derivative of (1.6),

$$
\frac{\partial F_{x}}{\partial x^{\omega}}=0
$$


if in these latter equations $\partial v_{u} / \partial x^{\mu}$ is replaced by $W_{\mu \imath} .\left(1.6^{\prime}\right)$ is then and only then identically satisfied, when (2.1) is an algebraic consequence of (1.6). In the same way $\left(1.4^{\prime \prime}\right)$ and $\left(1.6^{\prime \prime}\right)$ are algebraic consequences of (1.6), (2.1) and

$$
\frac{\partial}{\partial x^{\frac{F}{5}}} \frac{\partial F_{x}}{\partial x^{\omega}}=0
$$

if in this latter equation all derivatives $\partial v_{u} / \partial x^{\mu}$ are replaced by $W_{\mu u}$ and $\left(1.6^{\prime \prime}\right)$ is identically satisfied when (2.2) is an algebraic consequence of (1.6) and (2.1). The process comes to an end when one of the equations derived from (1.6) by differentiation is an algebraic consequence of all preceding ones, or, when the number of independent equations between the $v_{u}$ becomes greater than $N$.

Considering the solution of (1.4) in this way we are now able to put the condition of integrability of the covariant equation (1.1) equivalent to (1.4) into a covariant form. (1.6) is equivalent to

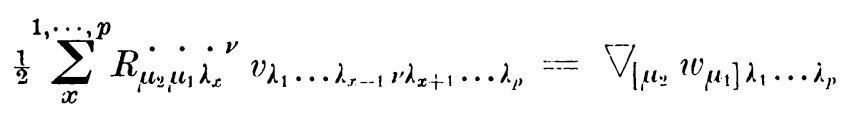

when on the right side always first derivatives of $v_{\lambda_{1}} \ldots \lambda_{p}$, are eliminated by means of (1.1). If we make use of the quantity

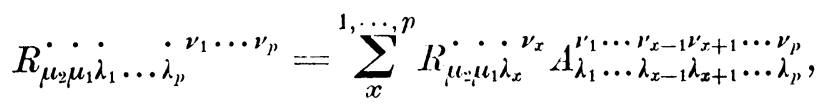

(2.3) can be written

$$
\frac{1}{2} R_{\mu_{\mu} \mu_{1} \dot{\lambda}_{1} \ldots \dot{\lambda}_{p}}^{\cdots{ }^{\prime \prime} \ldots \nu_{p}} v_{\nu_{1} \ldots \nu_{p}}=\nabla_{\left[\mu_{2}\right.} w_{\left.\mu_{1}\right]_{1}, \ldots \lambda_{p},} .
$$

The present investigation requiring a great many of suffixes we will use an abridged notation, writing $\lambda_{p}$ instead of $\lambda_{p} \ldots \lambda_{q+1}, \lambda_{q}$ being the next suffix of the $\lambda$-series occurring explicitly in the same term. So $v_{\lambda_{p}}$ stands for $v_{\lambda_{p} \ldots \lambda_{1}}, v_{\lambda_{p}} w_{\lambda_{q}}(p>q)$ for $v_{\lambda_{p} \ldots \lambda_{q+1}} w_{\lambda_{q} \ldots \lambda_{1}}, v_{\lambda_{p}} w_{\lambda_{q}}(p<q)$ for $v_{\lambda_{p}} \ldots \lambda_{1}$ $w_{\lambda_{q} \ldots \lambda_{p+1}}$, and $\nabla_{\lambda_{p}} w_{\lambda_{q}}(p>q)$ for $\nabla_{\lambda_{p} \ldots \lambda_{q}+1} w_{\lambda_{q} \ldots \lambda_{1}}=\nabla_{\lambda_{p}} \nabla_{\lambda_{p-1}} \ldots \nabla_{\lambda_{q}+1} w_{\lambda_{q} \ldots \lambda_{1}}$. This convention will be followed throughout this paper. Then (2.5) becomes

$$
\frac{1}{2} R_{\mu_{2} \lambda_{p}}^{\cdot}{ }^{v_{p}} v_{\nu_{p}}=\nabla_{\left[\mu_{2}\right.} w_{\left.\mu_{1}\right] \lambda_{p}} .
$$

By covariant differentiation of (2.6), always eliminating first derivatives of $v_{\mu \lambda_{p}}$, we get an infinite series of equations of the form 


$$
\stackrel{y}{S_{\mu_{y} \lambda_{p}}}+\stackrel{y}{S_{\mu_{y} \lambda_{p}}^{\cdot}}{ }^{\nu_{p}} v_{\nu_{p}}=0 \quad(y=2,3, \cdots),
$$

where the quantities $S$ are functions of $x^{\nu}$ and $v_{\nu_{p}}$, not containing derivatives of $v_{\nu_{p}}$. The first of these equations is (2.6) written in another way. Hence this equation is equivalent to (1.6). The first and second equations (2.7) are equivalent to (1.6) and (2.1), the first three equations (2.7) are equivalent to (1.6), (2.1) and (2.2), etc. The equations (2.7) containing only $v_{\lambda_{p}}$ and no derivatives, either are inconsistent, or there exists a number $q$, so that $\left(2.7_{q+1}\right)$ is an algebraic consequence of the preceding equations. Then the same holds for $\left(2.7_{q+2}\right)$ etc.

We have therefore proved the following theorem:

The first condition of integrability of the equation

$$
\nabla_{\mu} v_{\lambda_{p}}=w_{\mu \lambda_{p}}
$$

where $w_{\mu \lambda_{p}}$ contains only $x^{\nu}$ and $v_{\lambda_{p}}$ and no derivatives, is found by covariant differentiation and alternation of $(A)$ :

$$
\frac{1}{2} R_{\mu_{2} \lambda_{p}}^{\cdot \nu_{p}} v_{\nu_{p}}=\nabla_{\left[\mu_{2}\right.} w_{\left.\mu_{1}\right] \lambda_{p}}
$$

The other conditions are found by covariant differentiation of $\left(B_{1}\right)$, eliminating every time all derivatives of $v_{\lambda_{p}}$ :

$$
\left(B_{y-1}\right)
$$

$$
\stackrel{y}{S}_{\mu_{y} \lambda_{p}}+\stackrel{y}{S_{\mu_{y} \lambda_{p}}^{\cdot}} \nu_{\nu_{p}}^{v_{p}} v_{\nu_{p}}=0 \quad(y=3,4, \cdots) .
$$

The quantities $S$ contain only $x^{\nu}$ and $v_{\lambda_{p}}$ and no derivatives of $v_{\lambda_{p}}$. Either the system $(B)$ is inconsistent, or there exists a number $q$, such that $\left(B_{q+1}\right)$ is an algebraic consequence of $\left(B_{1}, \cdots, B_{q}\right)$. In the first case $(A)$ admits no solution; in the second case $\left(B_{q+2}\right)$ etc. also are algebraic consequences of $\left(B_{1}, \cdots, B_{q}\right)$ and the general solution of $(A)$ depends on a finite number of arbitrary constants.

3. The linear case. When $w_{\mu \lambda_{p}}$ contains $v_{\lambda_{p}}$ only linearly, (1.1) takes the form*

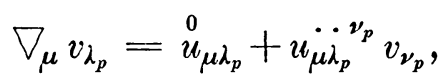

$\stackrel{0}{u}_{\mu \lambda_{p}}$ and $\ddot{u_{\mu \lambda_{p}}} \nu^{\nu_{p}}$ containing only $x^{\nu}$ and neither $v_{\lambda_{p}}$ nor derivatives of $v_{\lambda_{p}}$. The conditions of integrability are

* The case in which the right side of (3.1) reduces to zero is treated by Veblen and Thomas, these Transactions, vol. 25 (1923), pp. $584 \mathrm{ff}$. 


$$
\stackrel{0}{T}_{\mu_{y} \lambda_{p}}+T_{\mu_{v} i^{2}}^{\cdot \nu_{p}} v_{\nu_{p}}=0 \quad(y=2,3, \cdots),
$$

where

$$
\begin{aligned}
& \stackrel{0}{T}_{\mu_{2} \lambda_{p}}=-\nabla_{\left[\mu_{2}\right.}{\stackrel{0}{\left.u_{\mu_{1}}\right] \lambda_{p}}}-u_{\left[\mu_{1}\left|\dot{\lambda}_{p}\right|\right.}{ }^{v_{p}}{\stackrel{0}{\left.u_{\mu_{s}}\right] v_{p}},}, \\
& \stackrel{0}{T}_{\mu_{y+1} \lambda_{p}}==\nabla_{\mu_{y+1}}{\stackrel{0}{T_{\mu_{y} \lambda_{p}}}}+T_{\mu_{y} \lambda_{p}}^{\cdot} \cdot \stackrel{\nu}{\nu p}_{u_{\mu}}^{0}{ }_{\mu_{y+1}{ }^{\prime} p}
\end{aligned}
$$

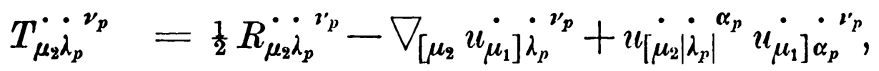

$$
\begin{aligned}
& T_{\mu_{y+1} \dot{\lambda}_{p}}^{\cdot \nu_{p}}=\nabla_{\mu_{y+1}} T_{\mu_{y} \lambda_{p}}^{\cdot \nu_{p}}+T_{\mu_{y} \lambda_{p}}^{\cdot \alpha_{p}} u_{\mu_{y+1} \alpha_{p}}^{\cdot \nu_{p}} \quad(y=2,3, \ldots) \text {. }
\end{aligned}
$$

Now we assume that the first $q$ equations (3.2) admit a solution ${\stackrel{0}{v_{p}}}_{\text {. }}$. Then every solution of these equations has the form

$$
v_{\lambda_{p}}=\stackrel{0}{v_{\lambda_{p}}}+\underset{1}{\alpha} \stackrel{1}{v_{\lambda_{p}}}+\cdots+\underset{s}{\alpha} \stackrel{s}{v_{\lambda_{p}}}
$$

where $\underset{1}{\alpha}, \ldots, \underset{s}{\alpha}$ are arbitrary parameters, functions of $x^{\nu}$, and where

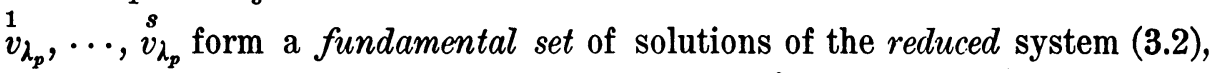
viz. the system deduced from (3.2) by setting $\stackrel{0}{u}_{\mu \lambda_{p}}=0$. In the reduced system all quantities $T$ with a suffix 0 vanish. Further we assume that the $(q+1)$ th equation (3.2) is an an algebraic consequence of the preceding oncs. When the system (3.2) is algebraically consistent there certainly will be a number $q$ with these properties.

Replacing $v_{\lambda_{p}}$ by ${\stackrel{0}{v_{p}}}_{\lambda_{p}}$ in the first $q$ equations (3.2) we have by differentiation

$$
\nabla_{\omega} \stackrel{0}{T}_{\mu_{y} \lambda_{p}}+\left(\nabla_{\omega} T_{\mu_{y} \lambda_{p}}^{\cdot \nu_{p}}\right){\stackrel{0}{v_{\nu}}}^{0}+T_{\mu_{y} \lambda_{p}}^{\cdot \nu_{p}} \nabla_{\omega}{\stackrel{0}{v_{\nu}}}^{2}=0 \quad(y=2, \cdots, q+1)
$$

Subtracting these equations from the second to the $(q+1)$ th equations $(3.2)$ (inclusive), we have, by (3.3),

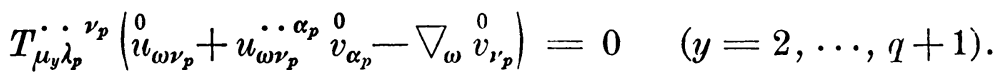

Hence

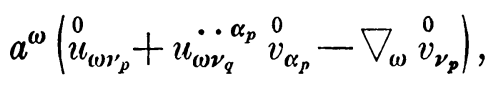

where $a^{\omega}$ is any arbitrary vector, is a solution of the reduced equation (3.2). Therefore, $v_{\lambda_{p}}, \ldots, v_{\lambda_{p}}$ being a fundamental set of solutions of the reduced equations (3.2), there must exist an equation of the form

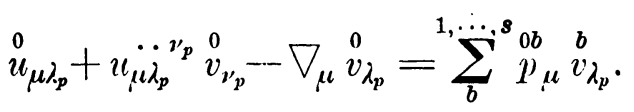


In the same way we prove, putting $\stackrel{a}{v}_{\lambda_{p}}, a=1, \ldots, s$, in the reduced equations (3.2), the existence of equations of the form

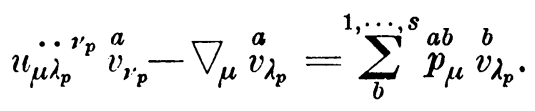

By differentiation and alternation of $(3.7,8)$ we obtain

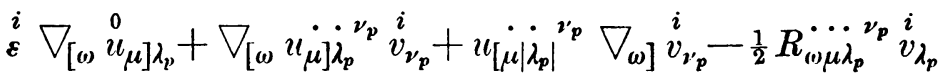

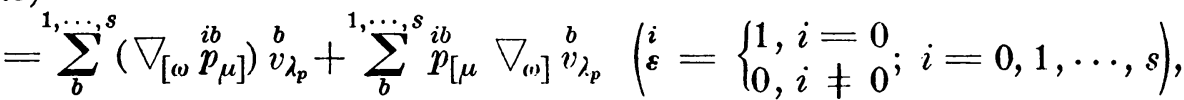

and these equations pass by means of $(3.2,3,7,8)$ into

$$
\nabla_{[\omega} \stackrel{i a}{p}_{\mu]}+\sum_{b} \stackrel{i b}{p}_{[\omega}^{b a} \stackrel{p}{p}_{\mu]}=0 \quad(i=0,1, \ldots, s)
$$

Now we will prove that the general solution of (3.1) has the form (3.4), and that the parameters $\underset{1}{\alpha}, \ldots, \underset{s}{\alpha}$ can be found by the integration of a completely integrable system. In order that (3.1) be satisfied by (3.4) it is necessary and sufficient that

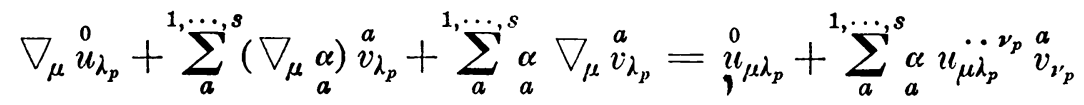

$$
+u \ddot{\mu \lambda_{p}} \nu_{p} \stackrel{0}{v}_{\nu_{p}^{\prime}},
$$

or, because of $(3.7,8)$,

$$
-\sum_{b}^{1, \ldots, s}{ }^{0 b} p_{\mu}{\stackrel{b}{v_{\lambda_{p}}}}^{b}+\sum_{a}^{1, \ldots, s}\left(\nabla_{\mu} \underset{a}{\alpha)} \stackrel{a}{v_{\lambda_{p}}}-\sum_{a, b}^{1, \ldots, s} \underset{a}{\alpha} \stackrel{a b}{p}{ }_{\mu}^{b}{\stackrel{b}{\lambda_{p}}}^{b}=0\right.
$$

or

$$
\nabla_{\lambda} \underset{a}{\alpha}=\stackrel{0 a}{p_{\lambda}}+\sum_{b}^{1, \cdots,} \underset{b}{s} \underset{b}{b a}
$$

The conditions of integrability of (3.13) are

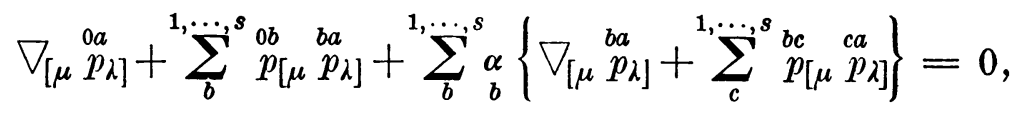

and these equations are identically satisfied in consequence of (3.10).

Hence, the integration of (3.1) is reduced to the algebraic solution of (3.2) and the integration of the completely integrable system (3.13). In the following 
sections of this chapter we give some applications to problems of differential geometry.

4. Conformal transformations of a $V_{n} *^{*}$ The equation

$$
{ }^{\prime} g_{\lambda \mu}=\sigma g_{\lambda \mu}
$$

gives a conformal transformation of a $V_{n}$ with fundamental tensor $g_{\lambda \mu}$. The transformed curvature affinor is

$$
\begin{aligned}
{ }^{\prime} K_{\omega \mu \lambda}^{\cdots \nu} & =K_{(\omega \mu \lambda}^{\cdots \nu}-g_{[\omega[\lambda} s_{\mu] \alpha]} g^{\alpha \nu} \\
s_{\mu \lambda} & =2 \nabla_{\mu} s_{\lambda}-s_{\mu} s_{\lambda}+\frac{1}{2} s_{\omega} s^{\alpha} g_{\mu \lambda}, \\
s_{\lambda} & =\nabla_{\lambda} \log \sigma .
\end{aligned}
$$

The transformation of $K_{\mu \lambda}=K_{\alpha \mu \lambda}^{\cdots \alpha}$ is given by

$$
{ }^{\prime} K_{\mu \lambda}=K_{\mu \lambda}+\frac{1}{4}\left\{(n-2) s_{\mu \lambda}+s_{\alpha \beta} g^{\alpha \beta} g_{\mu \lambda}\right\} \text {. }
$$

If $K_{\mu \lambda}$ vanishes the $V_{n}$ is often called an "Finstein space". The transformation of the tensor $L_{\mu \lambda}$,

$$
\begin{aligned}
& L_{\mu \lambda}=-K_{\mu \lambda}+\frac{1}{2(n-1)} K g_{\mu \lambda} ; \quad K=K_{\alpha \beta} g^{\alpha \beta}, \\
& K_{\mu \lambda}=-L_{\mu \lambda}-\frac{1}{n-2} L g_{\mu \lambda} ; \quad L=L_{\alpha \beta} g^{\alpha \beta},
\end{aligned}
$$

is more simple:

$$
\frac{4}{n-2}{ }^{\prime} L_{\mu \lambda}=\frac{4}{n-2} L_{\mu \lambda}-s_{\mu \lambda}
$$

It is well known that the $V_{n}$ can then and only then be transformed conformally into an $\boldsymbol{R}_{n}$ if the conformal curvature affinor defined by

$$
C_{\omega \mu \lambda \nu}=K_{\omega \mu \lambda \nu}-\frac{4}{n-2} g_{[\omega[\lambda} L_{\mu] \nu]}
$$

vanishes.t

Now we will deduce the necessary and sufficient conditions in order that a $V_{n}$ can be transformed conformally into an Einstein space. By (4.7) we have

$$
2 \nabla_{\mu} s_{\lambda}-s_{\mu} s_{\lambda}+\frac{1}{2} s_{\alpha} s^{\alpha} g_{\mu \lambda}=\frac{4}{n-2} L_{\mu \lambda},
$$

an equation of the form (1.1).

${ }^{*}$ Cf. for the formulae (4.1-8), R. K., pp. $168 \mathrm{ff}$.

$\dagger$ Mathematische Zeitschrift, vol. 11 (1921), p. 83. 
The first condition of integrability is

$$
K_{\omega \mu \lambda}^{\cdots \nu} s_{\nu}-s_{[\mu}\left\{-\frac{1}{4} g_{\omega] \lambda} s_{\alpha} s^{\alpha}+\frac{2}{n-2} L_{\omega] \lambda}\right\}
$$

$$
+s^{\alpha} g_{\lambda[\mu}\left\{\frac{1}{2} s_{\omega]} s_{\alpha}-\frac{1}{4} g_{\omega] \alpha \alpha} s_{\beta} s^{\beta}+\frac{2}{n-2} L_{\omega] \alpha}\right\}=\frac{4}{n-2} \nabla_{[\omega} L_{\mu] \lambda},
$$

or, because of (4.8),

$$
C_{\omega \mu \lambda}^{\cdots \nu} s_{\nu}=\frac{4}{n-2} \nabla_{[\omega} L_{\mu] \lambda} .
$$

By differentiation, using (4.9) to eliminate $\nabla_{\mu} s_{\lambda}$ we get the second condition

$$
\begin{gathered}
\left(\nabla_{[\xi} C_{\omega \mu \lambda]}^{\cdots \nu}\right) s_{\nu}+C_{\omega \mu \lambda}^{\cdots \nu}\left(\frac{1}{2} s_{\xi} s_{\nu}-\frac{1}{4} s_{\alpha} s^{\alpha} g_{\xi \nu}+\frac{2}{n-2} L_{\xi \nu}\right) \\
=\frac{4}{n-2} \nabla_{\xi} \nabla_{[\omega} L_{\mu] \lambda} .
\end{gathered}
$$

Proceeding in this way we obtain an infinite series of equations. Now these equations may be inconsistent, (4.9) admitting no solution. Or there exists a number $q$, such that the $(q+1)$ th equation is an algebraic consequence of the preceding ones. Then the same holds for all higher equations and (4.9) admits a general solution depending on a finite number of arbitrary constants.

We may also investigate the possibility of conformal transformations leaving $K_{\mu \lambda}$ invariant. Then $L_{\mu \lambda}$ is likewise invariant and (4.9) is reduced to

$$
2 \nabla_{\mu} s_{\lambda}-s_{\mu} s_{\lambda}+\frac{1}{2} s_{\alpha} s^{\alpha} g_{\mu \lambda}=0 .
$$

Introducing the vector

$$
s_{\lambda}^{\prime}=s^{-2} s_{\lambda} ; \quad s^{2}=s_{\alpha} s^{\alpha},
$$

(4.13) passes into the simpler equation

$$
\nabla_{\mu} s_{\lambda}^{\prime}=-\frac{1}{4} g_{\mu \lambda},
$$

of the form (3.1).

The first condition of integrability $f(4.15)$ is

$$
K_{\omega \mu \lambda}^{\cdots \nu} s_{\nu}^{\prime}=0 .
$$

The other conditions are 


$$
\begin{array}{ll}
\left(\begin{array}{lll}
\nabla_{\xi} & K_{\omega \mu \lambda}^{\cdots \nu}
\end{array}\right) s_{\nu}^{\prime}-\frac{1}{4} K_{\omega \mu \lambda \xi} & =0, \\
\left(\nabla_{\eta \xi} K_{\omega \mu \lambda}^{\cdots \cdots \nu}\right) s_{\nu}^{\prime}-\frac{1}{4} \nabla_{\xi} K_{\omega \mu \lambda \eta}-\frac{1}{4} \nabla_{\eta} K_{\omega \mu \lambda \xi} & =0, \\
\left(\nabla_{\zeta \eta \xi} K_{\omega \mu \lambda}^{\cdots \nu}\right) s_{\nu}^{\prime}-\frac{1}{4} \nabla_{\eta \xi} K_{\omega \mu \lambda \zeta}-\frac{1}{4} \nabla_{\zeta \xi} K_{\omega \mu \lambda \eta}-\frac{1}{4} \nabla_{\zeta \eta} K_{\omega \mu \lambda \xi \xi} & =0,
\end{array}
$$

etc. The conclusions are drawn in the same way as in the earlier case. We remark that for $n>2, g_{[\omega[\lambda} s_{\mu] \alpha]}$ vanishes only when $s_{\mu \lambda}=0$. Hence by (4.2) the deduced conditions are for $n>2$ also necessary and sufficient in order that the conformal transformation leaves $K_{\omega \mu \lambda}^{\cdots{ }^{\nu}}$ invariant.

5. Geodesic transformations of an $A_{n} .{ }^{*}$ Every geodesic transformation, viz. a transformation of the $\Gamma_{\lambda \mu}^{\nu}$ leaving invariant the geodesic lines, has the form

$$
{ }^{\prime} \Gamma_{\lambda \mu}^{\nu}=\Gamma_{\lambda \mu}^{\nu}+A_{\lambda}^{\nu} p_{\mu}+A_{\mu}^{\nu} p_{\lambda},
$$

$p_{\lambda}$ being an arbitrary vector, function of $x^{\nu}$. The transformed curvature affinor is given by

$$
{ }^{\prime} R_{\omega \mu \lambda}^{\cdots \nu}=R_{\omega \mu \lambda}^{\cdots \nu}-2 p_{[\omega \mu]} A_{\lambda}^{\nu}+2 A_{[\omega}^{\prime \prime} p_{\mu] \lambda},
$$

where

$$
p_{\mu \lambda}=\nabla_{\mu} p_{\lambda}-p_{\mu} p_{\lambda}
$$

The transformation of $R_{\mu \lambda}=R_{\alpha \mu \lambda}^{\cdots \alpha}$ is given by

$$
{ }^{\prime} R_{\mu \lambda}=R_{\mu \lambda}+n p_{\mu \lambda}-p_{\lambda \mu} .
$$

The transformation of the tensor $P_{\mu \lambda}$ :

is more simple:

$$
\begin{aligned}
-\left(n^{2}-1\right) P_{\mu \lambda} & =n R_{\mu \lambda}+R_{\lambda \mu}, \\
R_{\mu \lambda} & =-n P_{\mu \lambda}+P_{\lambda \mu},
\end{aligned}
$$

$$
{ }^{\prime} P_{\mu \lambda}=P_{\mu \lambda}-p_{\mu \lambda} .
$$

It is well known that the $A_{n}$ can then and only then be transformed geodesically into an $E_{n}$, if the projective curvature affinor, defined by

\section{vanishes.t}

$$
P_{\omega \mu \lambda}^{\cdots \nu}=R_{\omega \mu \lambda}^{\cdots \nu}-2 P_{[\omega \mu]} A_{\lambda}^{\nu}+2 A_{[\omega}^{\nu} P_{\mu] \lambda},
$$

${ }^{*}$ Cf. for the formulae (5.1-7), R. K., pp. 129 ff.

† Weyl, Göttinger Nachrichten (1921), pp. 99-112. 
First we will deduce the necessary and sufficient conditions in order that an $A_{n}$ can be transformed conformally into an $A_{n}$ with $R_{\mu \lambda}=0$. By (5.3) and (5.6) we have

$$
\nabla_{\mu} p_{\lambda}-p_{\mu} p_{\lambda}=P_{\mu \lambda}
$$

an equation of the form (1.1).

The first condition of integrability is

$$
\frac{1}{2} h_{\omega \mu \lambda}^{\prime \nu} p_{\nu}-P_{[\omega \mu]} \mu_{\lambda}-p_{[\mu} P_{\omega] \lambda}=\nabla_{[\omega} P_{\mu] \lambda}
$$

or, because of (5.7),

$$
\stackrel{P_{(1)}^{\prime} \mu \lambda^{\nu} p^{\prime} \nu^{\prime}}{\cdots}=2 \nabla_{[(1)} P_{\mu] \lambda}
$$

By differentiation, using (5.8) to eliminate $\nabla_{\mu} p_{\lambda}$, we get the second equation,

$$
\left(\nabla_{\xi} P_{(1) \mu \lambda}^{\cdots \nu}\right) p_{\nu}+P_{(1) \mu \lambda}^{\cdots \nu}\left(p_{\xi} p_{\nu}+P_{\xi \nu}\right)=2 \nabla_{\xi} \nabla_{[\omega} P_{\mu] \lambda} .
$$

Proceeding in this way we obtain an infinite series of equations. The conclusions are drawn in the same way as in the preceding section.

Secondly we consider the possibility of geodesic transformations leaving $R_{\mu \lambda}$ invariant. Then $P_{\mu \lambda}$ is also invariant and (5.8) passes into

$$
\nabla_{\mu} p_{\lambda}-p_{\mu} p_{\lambda}=0
$$

In consequence of this equation $p_{\lambda}$ is a gradient vector:

Introducing the vector

$$
p_{\lambda}=\nabla_{\lambda} p
$$

$$
p_{\lambda}^{\prime}=e^{-p} p_{\lambda}
$$

(5.12) passes into the simpler equation

$$
\nabla_{\mu} p_{\lambda}^{\prime}=0
$$

of the form (3.1).

Hence, a "eorlesic transformation leaving invariant $R_{\mu \lambda}$ exists only in an $A_{n}$ admitting a constant vector. The first condition of integrability of (5.15) is

$$
R_{(\omega) \mu \lambda}^{\cdots \prime} p_{\lambda}^{\prime}=0
$$


The other conditions are*

$$
\begin{aligned}
& \left(\nabla_{\xi} R_{\omega \mu \lambda}^{\cdots \nu}\right) p_{\lambda}^{\prime}=0 \\
& \left(\nabla_{\eta \xi} R_{\omega \mu \lambda}^{\cdots \nu}\right) p_{\lambda}^{\prime}=0, \text { etc. }
\end{aligned}
$$

The conclusions are drawn in the same way as in the earlier cases. We remark that $p_{[\omega \mu]} A_{\lambda}^{\nu}+A_{[\omega}^{\nu^{\prime}} p_{\mu] \lambda}$ vanishes only when $p_{\mu \lambda}=0$. Hence by (5.2) the deduced conditions are also necessary and sufficient in order that the geodesic tranformation leaves $R_{\omega \mu \lambda}^{\cdots \nu}$ invariant.

B. ON COVARIANT EQUATIONS WHICH ARE LINEAR HOMOGENEOUS IN THE FIRST COVARIANT DERIVATIVES

6. Properties of permutational operators. We consider systems of equations of the form

$$
\stackrel{\xi}{P} \nabla_{\mu} v_{\lambda_{p}}=\stackrel{\xi}{w}_{\mu \lambda_{p}} \quad(\xi=1, \cdots, z)
$$

where the quantity $w_{\mu \lambda_{p}}=w_{\mu \lambda_{p} \ldots \lambda_{1}}$ is a function of $x^{\nu}$ and $v_{\nu_{p}}=v_{\nu_{p}} \ldots \nu_{1}$ only and does not contain derivatives of $v_{\nu_{p}}$, and where the operators $\stackrel{\xi}{P}$ are homogeneous linear functions of the $(p+1)$ ! permutations of the $p+1$ suffixes $\mu \lambda_{p} \ldots \lambda_{1}$ with constant coefficients. So for $p=2$ a system of this form is, for example,

$$
\begin{aligned}
a \nabla_{\mu} v_{x \lambda}+b \nabla_{x} v_{\mu \lambda}+c \nabla_{\lambda} v_{x \mu} & =\stackrel{1}{w_{\mu x \lambda},} \\
e \nabla_{\mu} v_{\lambda x}+f \nabla_{\lambda} v_{\mu x} & =\stackrel{2}{w}_{\mu x \lambda} .
\end{aligned}
$$

First we have to deduce some purely algebraic properties of the operators $P . t$ These operators form an associative algebra with $(p+1)$ ! units, which is composed of $k$ different subalgebras with $\varepsilon_{1}^{2}, \cdots, \varepsilon_{k}^{2}$ units, $\varepsilon_{1}^{2}+\cdots+\varepsilon_{k}^{2}=(p+1)$ !; $k$ is the number of different solutions of the equation $x+y+z+\cdots=p+1$; $x, y, z$ being positive integers. E. g., for $p=5, k=11$ and the subalgebras have $1,25,81,100,25,256,100,25,81,25,1$ numbers, respectively. The numbers of two different subalgebras annihilate each other by multiplication, and the units $e_{u v}^{i}$ of any subalgebra with $\varepsilon_{i}^{2}$ units may be chosen in such a way that

$$
\stackrel{i}{e_{u v}} \stackrel{i}{e_{w x}}=\left\{\begin{array}{c}
e_{u x}, v=w \\
0, v \neq w
\end{array}, \quad u, v, v, x=1, \ldots, \varepsilon_{i} .\right.
$$

- 'The conditions (5.17) were first deduced by Eisenhart and Veblen, Proceedings of the National Academy of Sciences, vol. 8 (1922), pp. 19-23.

†Cf. R. K., Abschn. VII. 
The number $k$ and the different subalgebras are uniquely determined, and the same holds for the $k$ sums $\sum_{u} e_{u u}^{i}$ of the idempotent numbers $e_{u u}^{i}$. These sums are the moduli of the subalgebras, their sum being the modulus $I$ of the algebra itself. The numbers $e_{u u}$ are called chief-units. They are not uniquely determined. They form a chief-series of the subalgebra and this chief-series can be chosen in an infinite number of ways. A chiefseries of the algebra is built up by $k$ chief-series of the $k$ subalgebras, each chief-series chosen in an arbitrary way.

The rules of multiplication of the numbers of a subalgebra are the same as the rules of first transvection (erste Uberschiebung) of the mixed affinors of the second degree in $\varepsilon_{i}$ variables. Hence the operator $P$ can be written*

$$
\sum_{i} P_{\alpha_{i}}^{\cdot \gamma_{i}} ; \quad{ }_{i}, \gamma_{i}=1, \ldots, \varepsilon_{i} ; \quad i=1, \ldots, k
$$

or, briefly, $P_{\alpha}^{\cdot \gamma}$, where $a$ and $\gamma$ take only values from $1, \cdots, N, N=\sum_{i} \varepsilon_{i}$, belonging to the same subalgebra. With this notation the properties of the operators $P$ can be easily deduced from the well known properties of the affinors $P_{\alpha}^{\cdot \gamma \prime}$ in a point of an $E_{N}$.

$N$ linearly independent contravariant vectors $e_{\alpha}^{\gamma}$ of the $E_{N}$ being given, every affinor $P_{\alpha}^{\cdot \gamma}$ can be written in one and only one way in the form

$$
P_{\alpha}^{\cdot \gamma}=\stackrel{\beta}{u_{\alpha}} \underset{\beta}{e^{\gamma}}
$$

From the vectors $u_{\alpha}$ there will be some, say $r$, linearly independent. If we denote them by $\stackrel{u}{p}_{\alpha}, u=1, \cdots, r$, there exist $r$ vectors $\frac{q^{\gamma}}{u}$, such that

$$
P_{\alpha}^{\cdot \gamma}=\sum_{u}^{1, \ldots, r} p_{\alpha} q_{u}^{\gamma}
$$

$r$ is the rank of $P$; this number is the sum of the subranks $r_{1}, \cdots, r_{k}$, belonging to the $k$ subalgebras. $r_{1}, \cdots, r_{k}$ are invariants of $P$. So are the $E_{r}$ and the $E_{n-r}$ determined by the alternate products $\frac{q_{1}^{\left[\gamma_{1}\right.}}{P} \cdots q_{r}^{\left.q_{r}\right]}$ and

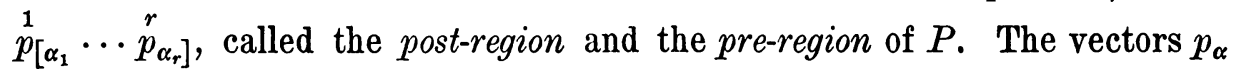
and $q^{\gamma}$ are not uniquely determined, but the set $p_{\alpha}$ is, when the set $q^{\gamma}$ is given in $E_{r}$, and vice versa.

\footnotetext{
${ }^{*}$ In this formula $\alpha_{i}$ and $\gamma_{i}$ do not stand for $\alpha_{i} \cdots \epsilon_{1}$ and $\gamma_{i} \cdots \gamma_{1}$.
} 
The operator $P$ is then and only then idempotent when, for some choice of the set $p_{\alpha}, q^{\gamma}$,

$$
\stackrel{u}{p}_{c} q_{v}^{\alpha}=\left\{\begin{array}{l}
1, u=v \\
0, u \neq v
\end{array} ; \quad u, v=1, \cdots, r .\right.
$$

Then (6.7) holds for every choice of $p_{\alpha}$ or $q^{\gamma}$. To every idempotent operator $E$ belongs its conjugate operator $E=I-E$, which is also idempotent. The pre-region of $E$ is the post-region of $E^{\prime}$ and vice versa. Pre- and post-regions of an idempotent operator have no directions in common. $\quad P$ being idempotent, ${ }_{\alpha}^{u} \underset{u}{q}$ is a chief-unit for every $u$, hence an idempotent operator of rank $r$ is a sum of $r$ chief-units, $r_{1}$ from the first subalgebra, $r_{2}$ from the second, etc. The chief-units themselves are not uniquely determined, but the sums of the chief-units belonging to the same subalgebra are.

To every operator $P$ belongs an infinite number of idempotent operators having the same pre-(post-)region as $P$. Having written $P$ in the form (6.6), we have only to choose in an arbitrary way $N-r$ covariant vectors ${ }_{p}^{r+1}, \ldots, N_{\alpha}$ linearly independent of each other and of $\stackrel{1}{p}_{\alpha}, \ldots, \stackrel{r}{p}_{\alpha}$, and form the reciprocal system $p_{1}^{\gamma}, \cdots, p_{N}^{\gamma}$, so that

Then

$$
p_{\imath}^{\alpha} \stackrel{v}{p}_{p^{\prime}}=\left\{\begin{array}{l}
1, u=v \\
10, u \neq v
\end{array}\right.
$$

$$
E_{\alpha}^{\cdot \gamma}=\sum_{u}^{1, \cdots, r} p_{\alpha} p_{u}^{p^{\gamma}}
$$

is an idempotent operator with the same pre-region as $P$. In the same way we form an idempotent operator $F$, having the same post-region as $P$, using $N-r$ vectors $\underset{r+1}{q^{\gamma}}, \cdots, \underset{N}{q}$ and the reciprocal system $\stackrel{1}{q}_{\alpha}, \cdots, \stackrel{N}{q}_{\alpha}$. From the definition of $E$ and $F$ we have

and

$$
E P=P ; \quad P F=P,
$$

$$
\begin{aligned}
& E_{\alpha}^{\cdot \gamma}=\sum_{u}^{1, \cdots, N} P_{\alpha}^{\cdot \beta} \stackrel{u}{q}_{\beta} \underset{u}{p^{\gamma}}, \\
& F_{\alpha}^{\cdot \gamma}=\sum_{u}^{1, \cdots, N} \stackrel{u}{q}_{\alpha} \underset{u}{\beta} P_{\beta}^{\cdot \gamma} .
\end{aligned}
$$

Hence, $E$ and $F$ can be obtained from $P$ by post-(pre-)multiplication with an operator of rank $N$. 
Now we are able to consider the question when it is possible to write a quantity $w_{\mu \lambda_{p}}$ in the form

$$
w_{\mu \mu \lambda_{p}}=P u_{\mu \lambda_{p}} .
$$

Operating on (6.12) with $E$ we have, by (6.10),

An equivalent to (6.13) is

$$
\begin{aligned}
& E w_{\mu \lambda_{p}}=w_{\mu \lambda_{p}} . \\
& E^{\prime} w_{\mu \lambda_{p}}=0,
\end{aligned}
$$

$E_{1}^{\prime}$ being the conjugate to $E$. But, by using the reciprocal system $\stackrel{1}{q}_{\alpha}, \ldots, \stackrel{N}{q}_{\alpha}$ we have, in consequence of (6.13) and (6.11),

$$
\begin{aligned}
w_{\mu \lambda_{\rho}} & =E_{\alpha}^{\cdot \gamma^{\prime}} w_{\mu \lambda_{p}} \\
& =P_{\alpha}^{\cdot \beta^{1, \cdots \cdots}} \sum_{u}^{N^{N} q_{\beta}} \underset{u}{p^{\gamma}} w_{\mu \lambda_{p}} .
\end{aligned}
$$

Thus (6.13) or (6.14) is a necessary and sufficient condition that $w_{\mu \lambda_{p}}$ be a result of the operation $P$.

Hence, if $\stackrel{x}{E}$ is an idempotent operator, having the same pre-region as $\stackrel{i r}{P}$ in $(6.1)$, and $\stackrel{x}{E^{\prime}}$ is the conjugate operator,

$$
{\stackrel{\xi}{E^{\prime}}}^{\prime} \stackrel{\xi}{w}_{\mu \lambda_{p}}=0 \quad(\xi=1, \cdots, z)
$$

are the first conditions of integrability of the system (6.1).

We now prove the following theorem:

$A$ consistent system of equations of the form (6.1) is equivalent to only one equation of that form.*

We consider the first two equations of (6.1). If the ranks of $\stackrel{1}{P}$ and $\stackrel{2}{P}$ are $\stackrel{1}{r}$ and $\stackrel{2}{r}$, and if the post-regions of these operators have $s$ directions in common, these equations can be written in the form

$$
\begin{gathered}
\sum_{1}^{1} u \stackrel{u}{p}_{\alpha}{ }_{u}^{\gamma} \nabla_{\mu} v_{\lambda_{p}}=\stackrel{1}{w}_{\mu \lambda_{p}}, \\
\sum_{y+1}^{t} u \stackrel{u}{r}_{\alpha} \underset{u}{q} \nabla_{\mu} v_{\lambda_{p}}=\stackrel{2}{w}_{\mu \lambda_{p}}, \\
r-y=s, \quad t-y=\stackrel{2}{r} .
\end{gathered}
$$

*'The algebraic equivalent of this theorem was first deduced by Weyl, Rendiconti del Circolo Matematico di Palermo, vol. 48 (1924), p. 32. 
Completing the sets $p_{\alpha}, q^{\gamma}$ and $r_{\alpha}$ in an arbitrary way to sets of $N$ linearly independent vectors and forming the reciprocal sets $p^{\gamma}, q_{\alpha}$ and $r^{\gamma},(6.17)$ is equivalent to

$$
\begin{aligned}
& \sum_{1}^{r} u \stackrel{u}{q}_{\alpha} \underset{u}{q} \nabla_{\mu} v_{\lambda_{p}}=\sum_{1}^{N} u \stackrel{u}{q}_{\alpha} \underset{u}{p^{\gamma}} \stackrel{1}{w}_{\mu_{p}}, \\
& \sum_{y+1}^{t} u \stackrel{u}{q}_{\alpha} \underset{u}{q} \nabla_{\mu} v_{\lambda_{p}}=\sum_{1}^{N} \stackrel{u}{q}_{\alpha} \underset{u}{r^{\gamma}} \stackrel{2}{w}_{\mu \lambda_{p}} \text {. }
\end{aligned}
$$

In order that these equations be consistent it is necessary and sufficient that

$$
\begin{aligned}
& \stackrel{u}{q}_{\alpha} p_{u}^{\gamma} \stackrel{1}{w}_{\mu \lambda_{p}}=\stackrel{u}{q}_{\alpha} \nu_{u}^{\gamma^{\prime}} \stackrel{2}{w}_{\mu_{\lambda_{p}}} \quad(u=y+1, \ldots, \stackrel{1}{r}) ; \\
& \stackrel{u}{q}_{\alpha} p_{u}^{\gamma} \stackrel{1}{w}_{\mu \lambda_{p}}=0 \quad(u=\stackrel{1}{r}+1, \ldots, N) \text {; } \\
& \stackrel{u}{q}_{u} \underset{u}{r^{\gamma}} \stackrel{2}{w}_{\mu \lambda_{p}}=0 \quad(u=1, \cdots, y, t+1, \ldots, N) .
\end{aligned}
$$

In the case of consistency (6.18) is equivalent to

$$
\begin{aligned}
& \stackrel{u}{q}_{\alpha} \underset{u}{p^{\gamma}} \nabla_{\mu} v_{\lambda_{p}}=\stackrel{u}{q}_{\alpha} \underset{u}{p^{\gamma}} \stackrel{1}{w}_{w_{\lambda_{p}}} \quad(u=1, \ldots, r) ; \\
& \stackrel{u}{q}_{\alpha} \underset{u}{\gamma} \nabla_{\mu} v_{\lambda_{p}}=\stackrel{u}{q}_{\alpha} \underset{u}{r^{\gamma}} \stackrel{2}{w}_{w_{\mu \lambda_{p}}} \quad(u=\stackrel{1}{r}+1, \ldots, t),
\end{aligned}
$$

or

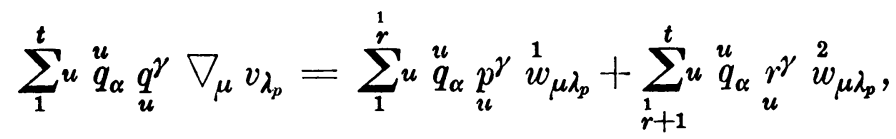

q. e. d. Proceeding in this way it can be proved that all equations (6.1) are equivalent to only one equation.

It may be remarked that the post-region of the operator in the left side of (6.21) is the smallest region that contains the post-regions of both $\stackrel{P}{P}$ and $\stackrel{2}{P}$. In the same way the operator in the left side of the equation equivalent to all equations (6.1) has a post-region that is the smallest one containing all post-regions of the operators $P$. We call this region the combined post-region of the operators $P$. The sum of the dimensions of the combined pre-region, constructed in the same way, and the combined post-region is in general not equal to $N$.

Suppose now that an algebraic equation is given of the form

$$
P u_{\mu} v_{\lambda_{p}}=w_{\mu \lambda_{\mu}} \text {, }
$$


$u_{\mu}$ and $w_{\mu \lambda_{p}}$ being known quantities. The left side of this equation can in one and only one manner be written in the form

$$
P u_{\mu} v_{\lambda_{p}}=\stackrel{1}{\varphi} u_{\mu} v_{\lambda_{p}}+\stackrel{2}{C} \stackrel{2}{\varphi} u_{\mu} v_{\lambda_{p}}+\cdots+\stackrel{p+1}{C} \stackrel{p+1}{\varphi} u_{\mu} v_{\lambda_{p}},
$$

where $\stackrel{1}{\varphi}, \ldots, \stackrel{p+1}{\varphi}$ operate only on $\lambda_{1}, \ldots, \lambda_{p}$ and $\stackrel{2}{C}, \ldots, \stackrel{p+1}{C}$ are the cyclical operators transforming $\mu \lambda_{1} \ldots \lambda_{p}$ into $\lambda_{1} \ldots \lambda_{p} \mu, \lambda_{2} \ldots \lambda_{p} \mu \lambda_{1}$, etc. The operators operating only on $\lambda_{1} \ldots \lambda_{p}$ form an associative algebra with $p$ ! units. In this algebra the operators $\stackrel{1}{\varphi}, \ldots, \stackrel{p+1}{\varphi}$ have a combined postregion and we can form an idempotent operator $U$ having this same postregion. Let $U^{\prime}$ be the conjugate operator of $U$. Then $x_{\lambda_{p}}$ being any quantity of degree $p$, we have identically

$$
P u_{\mu} U^{\prime} x_{\lambda_{p}}=0 \text {. }
$$

On the other hand every solution of $P u_{\mu} v_{\lambda_{p}}=0$ has the form $U^{\prime} x_{\lambda_{p}}$. Therefore, we have only to consider such solutions of (6.22) as satisfy the equation

$$
U v_{\lambda_{p}}=v_{\lambda_{p}}
$$

These solutions being known, every other solution can be found by adding $U^{\prime} x_{\lambda_{p}}, x_{\lambda_{p}}$ being an arbitrary quantity of degree $p$.

7. Determination of the characteristic number of a permutational operator. In the preceding section we have seen that every operator has a definite rank. Now we will determine the number of linearly independent components of a quantity $P v_{\lambda_{q}}, v_{\lambda_{q}}$ being an arbitrary quantity of degree $q$ and $P$ a homogeneous linear function of the $q l$ permutations of $\lambda_{1} \ldots \lambda_{q}$. We call this number the characteristic number of $P$ and denote it by $(P)$. In some simple cases the characteristic number can easily be found.* If for instance $P$ alternates (mixes) + the first $t$ suffixes, leaving the other suffixes unchanged, we have obviously

and

$$
(P)=\left(\begin{array}{c}
n \\
t
\end{array}\right) n^{q-t}
$$

* Comp. p. 459 , note *.

$\dagger$ By alternating we get for instance from $v_{x \lambda \mu}$

and by mixing

$$
v_{[x \lambda \mu]}=\frac{1}{3 !}\left(v_{x \lambda \mu}+v_{\lambda \mu x}+v_{\mu x \lambda}-v_{x \mu \lambda}-v_{\lambda x \mu}-v_{\mu \lambda x}\right)
$$

$$
v_{(x \lambda \mu)}=\frac{1}{3 !}\left(v_{x \lambda \mu}+v_{\lambda \mu x}+v_{\mu x \lambda}+v_{x \mu \lambda}+v_{\lambda x \mu}+v_{\mu \lambda x}\right) .
$$


respectively. In the general case it would be theoretically possible to determine the non-vanishing determinants of the matrix of the components of $P v_{\lambda_{q}}$. In practice this method is far too laborious. But we can find the desired number in a very easy way, making use of the author's development of a covariant quantity in a series of indivisible quantities, viz. quantities that have no linear covariant with a smaller number of components. These indivisible quantities correspond to the chief-units of the algebra of permutational operators, and in consequence it is possible to write any quantity as a sum of indivisible quantities, using an arbitrary chosen chief series $\stackrel{1}{I}, \ldots, \stackrel{n}{I}$. By using this chief-series we obtain the development

$$
P v_{\lambda_{q}}=(\stackrel{1}{I}+\cdots+\stackrel{N}{I}) P v_{\lambda_{q}} .
$$

Now among the $N$ indivisible quantities, obtained in this way, some may be zero, and others may be each other's covariants. If $r$ is the rank of $P$, just $r$ of them are not zero and independent. The characteristic number of one of the remaining $r$ operators $\stackrel{\alpha}{I}_{\alpha} P$ is the same as the characteristic number of $\stackrel{\alpha}{I}$, because $\stackrel{\alpha}{I} v_{\lambda_{q}}$ has no linear covariants with a smaller number of components. But $(\stackrel{\alpha}{I})$ can easily be found, because it is possible to choose the chief-series in a very simple way.* The process is shortened by the circumstance that all chief-units, belonging to the same subalgebra, have the same characteristic number. So we have only to determine the subranks $\stackrel{1}{r}, \ldots, \stackrel{k}{r}$ and the characteristic numbers belonging to each of the $k$ subalgebras.

The method just indicated can be considered from another point of view. The equation

being equivalent to

$$
\begin{aligned}
& P v_{\lambda_{q}}=0 \\
& F v_{\lambda_{q}}=0,
\end{aligned}
$$

where $F$ is an idempotent operator with the same post-region as $P,(P)$ and $(F)$ must be equal. Now $F$ can be written (p. 455) as a sum of chief-units, $\dagger$ and so we have only to determine the characteristic numbers of these chief-units. Each of the indivisible quantities belonging to these chief-units is a linear covariant of one of the terms $\stackrel{\alpha}{I} P v_{\lambda_{q}}$ obtained above, but the

*Also a general formula can be used deduced by I. Schur in an investigation on the theory of the linear homogeneous group (Dissert. 1901, p. 51). Comp. H. Weyl, Mathematische Zeitschrift, vol. 23 (1925), p. 300.

$\dagger$ These chief-units were first used by Weyl in an investigation concerning the theory of invariants, Rendiconti del Circolo Matematico di Palermo, vol. 48 (1924), pp. 29-36. 
chief-units contained in $F$ do not belong in general to the artificial chief-series we made use of. This way seems to be a shorter one, but in practice it is not so easy to determine the $r$ chief-units belonging to $F$ and so the use of the artificial chief-series must be recommended.

We give some examples, which will be used later on.

Example 1. Let ${ }_{x} A$ be an operator, alternating the first $x$ suffixes $\lambda_{q} \ldots \lambda_{q-x+1}$ and ${ }_{y+1} M, x+y=q$, an operator mixing $\lambda_{q}$ and the last $q-x$ suffixes $\lambda_{q-x}, \ldots, \lambda_{1}$. Then a number ${ }_{x} A{ }_{y+1} M$ is a chief-unit of an artificial chief-series as used above. Also ${ }_{y+1} M_{x} A$ is chief-unit of another artificial chiefseries, frequently used. For $q<6$ all chief-units of artificial chief-series have such a form, though there may be more than one region for alternating or mixing. ${ }^{*}$ The characteristic number of ${ }_{x} A{ }_{y+1} M$ and ${ }_{y+1} M_{x} A$ is

$$
\begin{aligned}
& \left({ }_{x} A{ }_{y+1} M\right)=\left({ }_{y+1} M{ }_{x} A\right) \\
= & \left(\begin{array}{c}
n \\
x-1
\end{array}\right)\left(\begin{array}{l}
n+y \\
y+1
\end{array}\right)-\left(\begin{array}{c}
n \\
x-2
\end{array}\right)\left(\begin{array}{c}
n+y+1 \\
y+2
\end{array}\right)+\cdots+(-1)^{x-1}\left(\begin{array}{c}
n+q-1 \\
q
\end{array}\right) .
\end{aligned}
$$

The first term in this series is $\left({ }_{x-1} A{ }_{y+1} M\right),{ }_{x-1} A$ alternating $x-1$ suffixes and ${ }_{y+1} M$ mixing the other $y+1$, the second is $\left({ }_{x-2} A_{y+2} M\right)$ etc.

Example 2. Let ${ }_{x} M$ be an operator mixing the first $x$ suffixes $\lambda_{q} \ldots \lambda_{q-x+1}$ and ${ }_{y+1} M$ the operator used in Example 1. By using the above mentioned second chief-series we find that $\left({ }_{x} M_{y+1} M\right)$ is equal to $\left({ }_{x} M_{y} M\right),{ }_{y} M$ mixing only $\lambda_{x+1}, \cdots, \lambda_{y}$. Hence we have

$$
\left({ }_{x} M_{y+1} M\right)=\left({ }_{x} M_{y} M\right)=\left(\begin{array}{c}
n+x-1 \\
x
\end{array}\right)\left(\begin{array}{c}
n+y-1 \\
y
\end{array}\right) .
$$

8. A theorem of Lie. Given a system of partial differential equations with $n$ independent variables $x^{\nu}$ and an arbitrary number of dependent ones. There are three cases:

1. The equations are inconsistent.

2. The general solution depends on a finite number of arbitrary constants.

3. The general solution contains arbitrary functions of $x^{\nu}$. An example of the first and second case is the equation

$$
\nabla_{\mu} v_{\lambda}=0
$$

having in general no solution and in special cases a general solution depending on a finite number of arbitrary constants. An example of the third case is the equation

$$
\nabla_{[\mu} v_{\lambda]}=0,
$$

${ }^{*}$ For $n \geqq 6$ at least one chief-unit in every subalgebra has such a form. 
having the general solution

$$
v_{\lambda}=\nabla_{\lambda} p
$$

$p$ being an arbitrary scalar function.

The equations being consistent, $\mathrm{Lie}^{*}$ has proved that the following conditions are necessary and sufficient for the second case:

1. There exists a number $s$, such that every derivative of order $s$ may be expressed as a function of the lower derivatives, and that the same does not hold for every derivative of order $s-1$.

2. By differentiating and eliminating the system can be put in such a form that, by differentiating all equations once, and eliminating all derivatives of order $s+1$, no equation can be obtained that is not a consequence of the equations of the system.

Let us inquire in which cases the first of Lie's conditions holds for an equation of the form

$$
P \nabla_{\mu} v_{\lambda_{p}}=w_{\mu \lambda_{p}}
$$

where $w_{\mu \lambda_{p}}$ is a function of $x^{\nu}$ and $v_{\lambda_{p}}$ only, not containing derivatives of $v_{\lambda_{p}}$. If $U$ is an idempotent operator in $\lambda_{1} \ldots \lambda_{p}$ only, belonging to $P$ as defined in $\S 6, U^{\prime}$ the conjugate of $U,{\stackrel{0}{\lambda_{p}}}_{\text {any }}$ andution of (8.4), and $s_{\lambda_{p}}$ an arbitrary quantity of degree $p$, then

$$
\stackrel{0}{v}_{\lambda_{p}}+U^{\prime} s_{\lambda_{p}}
$$

is also a solution of (8.4), because $P$ annihilates $U^{\prime}$. Hence the general solution of (8.4) depends for $U \neq I$ not on a finite number of arbitrary constants, when no other assumption is made. In consequence we consider only solutions of (8.4) satisfying the equation

$$
U v_{\lambda_{p}}=v_{\lambda_{p}}
$$

According to $\S 6$ the first condition of integrability of (8.4) is

$$
E^{\prime} w^{\prime} \mu \lambda_{p}=0,
$$

$E^{\prime}$ being the conjugate of an idempotent operator having the same preregion as $P$. This condition being satisfied, (8.4) can then and only then be solved for $\nabla_{\mu} v_{\lambda_{p}}$ when the number of components of the left side is equal to $n(U)$. Now this number is equal to $(P)$. Hence, if $(P)=n(U)$,

* Theorie der Transformationsgruppen, I, Chapter 10. 
(8.4) can be put into a form already considered in Chapter I. Supposing now $(P)<n(U)$ and differentiating (8.4) we have

$$
P \nabla_{\mu_{2}} v_{\lambda_{p}}=\nabla_{\mu_{2}} w_{\mu_{1} \lambda_{p}}
$$

The number of components of $P \nabla_{\mu_{\mathrm{z}}} v_{\lambda_{p}}$ and $\nabla_{\mu_{2}} v_{\lambda_{p}}$ being $n(P)$ and $n^{2}(U)$, (8.8) cannot be solved for $\nabla_{\mu_{2}} v_{\lambda_{p}}$. From (8.8) we obtain, however,

$$
P \nabla_{\left(\mu_{3}\right)} v_{\lambda_{p}}=\nabla_{\mu_{2}} w_{\mu_{1} \lambda_{p}}-\frac{1}{2} P R_{\mu_{2} i_{p}}^{\cdot \nu_{p}} v_{\nu_{p}}
$$

and in this equation the number of components of $P \nabla_{\left(\mu_{s}\right)} v_{\lambda_{p}}$ is equal to $\left(P_{2} M\right),{ }_{2} M$ being the operator mixing the suffixes $\mu_{2} \mu_{1}$. The number of components of $\nabla_{\left(\mu_{2}\right)} v_{\lambda_{p}}$ being $\left({ }_{2} M U\right)$, the solution of (8.9) for $\nabla_{\left(\mu_{2}\right)} v_{\lambda_{p}}$ is then and only then possible when $\left(P_{2} M\right)=\left({ }_{2} M U\right)$. In this case the right side of (8.9) containing only $v_{\lambda_{p}}$ and first derivatives, $\nabla_{\left(\mu_{z}\right)} v_{\lambda_{p}}$ and therefore also $\nabla_{\mu_{2}} v_{\lambda_{p}}$ can be expressed as a function of $v_{p_{p}}$ and first derivatives, so that the first of Lie's conditions is satisfied for $s=2$. When $\left.\left(P_{2} M\right)<{ }_{2} M U\right)$ we have, by differentiating (8.8),

$$
P \nabla_{\mu_{s}} v_{\lambda_{p}}=\nabla_{\mu_{s}} w_{\mu_{1} \lambda_{p}} \text {. }
$$

Now, because of the identity

$$
\begin{aligned}
p_{\mu_{3} \mu_{2} \mu_{1}}=p_{\left(\mu_{8} \mu_{2} \mu_{1}\right)} & -\frac{5}{8} p_{\left[\mu_{2} \mu_{8}\right] \mu_{1}}+\frac{1}{2} p_{\mu_{2}\left[\mu_{8} \mu_{1}\right]} \\
& +\frac{1}{6} p_{\left[\mu_{2} \mu_{1}\right] \mu_{3}}-\frac{5}{8} p_{\mu_{8}\left[\mu_{1} \mu_{2}\right]} \\
& -\frac{1}{2} p_{\mu_{1}\left[\mu_{2} \mu_{8}\right]}+\frac{1}{6} p_{\left[\mu_{8} \mu_{1}\right] \mu_{3}}{ }^{*}
\end{aligned}
$$

every quantity $p_{\mu_{s}}$ can be written as a sum of $p_{\left(\mu_{s}\right)}$ and several quantities all alternating in two neighboring suffixes. Making use of this property and bringing all terms alternating in two neighboring suffixes to the right, reducing them to first derivatives by means of $R_{\omega \mu \lambda}^{\cdots \nu}$, we obtain an equation of the form

$$
\begin{aligned}
P_{3} M \nabla_{\mu_{z}} v_{\lambda_{p}}=\nabla_{\mu_{s}} w_{\mu_{1} \lambda_{p}} & +\stackrel{3}{T_{\mu_{s}} \dot{\lambda}_{p}}{ }^{\nu_{p}} v_{\nu_{p}} \\
& +\stackrel{8}{T}_{\mu_{3} \dot{\lambda}_{p}}^{\alpha_{1} \nu_{p}} \nabla_{\alpha_{1}} v_{\nu_{p}}
\end{aligned}
$$

containing only derivatives up to the second on the right. In this way we can proceed, always reducing the difference of $\nabla_{\mu_{x}} v_{\lambda_{p}}$ and $\nabla_{\left(\mu_{x}\right)} v_{\lambda_{p}}$

* In this equation the abridged notation is not used. 
to derivatives of the order $x-2$ with the aid of $R_{\omega \mu \lambda}^{\cdots \nu}$. Then it may occur that for $x=q,\left(P_{q} M\right)=\left({ }_{q} M U\right)$. Then $\nabla_{\left(\mu_{q}\right)} v_{\lambda_{p}}$ and therefore $\nabla_{\mu_{q}} v_{\lambda_{p}}$ can be expressed as functions of the lower derivatives, so that the first of Lie's conditions is satisfied for $s=q$. In the other case such an expression can never be found and in the case of consistency (8.4) must have a general solution containing arbitrary functions.

We give some examples.

Example 1.

$$
\nabla_{[\mu} v_{\left.\lambda_{t}\right] \lambda_{p}}=w_{\mu \lambda_{p}} ; \quad t \leqq p
$$

$P$ is the operator alternating the suffixes $\mu \lambda_{1} \ldots \lambda_{t}$ and $U$ is the operator alternating $\lambda_{1} \ldots \lambda_{t}$. The characteristic numbers are, according to $\S 7$,

$$
\begin{aligned}
& \left(P_{x} M\right)=n^{p-t}\left\{\left(\begin{array}{l}
n \\
t
\end{array}\right)\left(\begin{array}{c}
n+x-1 \\
x
\end{array}\right)-\left(\begin{array}{c}
n \\
t-1
\end{array}\right)\left(\begin{array}{l}
n+x \\
x+1
\end{array}\right)+\cdots+(-1)^{t}\left(\begin{array}{c}
n+x+t-1 \\
t+x
\end{array}\right)\right\}, \\
& \left({ }_{x} M U\right)=n^{p-t}\left\{\left(\begin{array}{l}
n \\
t
\end{array}\right)\left(\begin{array}{c}
n+x-1 \\
x
\end{array}\right)\right\},
\end{aligned}
$$

so that always

$$
\left(P_{x} M\right)<\left({ }_{x} M U\right)
$$

Hence, (8.13) is inconsistent or the general solution contains arbitrary functions.

Example 2.

$$
\nabla_{(\mu} v_{\left.\lambda_{\varepsilon}\right) \lambda_{p}}=w_{\mu \lambda_{p}} ; \quad t \leqq p
$$

$P$ is the operator mixing the suffixes $\mu \lambda_{1} \ldots \lambda_{t}$ and $U$ mixes $\lambda_{1} \ldots \lambda_{t}$. The characteristic numbers are, according to $\S 7$,

$$
\begin{aligned}
& \left(P_{x} M\right)=n^{p-t}\left(\begin{array}{c}
n+t \\
t+1
\end{array}\right) \\
& \left(\begin{array}{c}
n+x-2 \\
x-1
\end{array}\right), \\
& \left.{ }_{x} M U\right)=n^{p-t}\left(\begin{array}{c}
n+t-1 \\
t
\end{array}\right)\left(\begin{array}{c}
n+x-1 \\
x
\end{array}\right) .
\end{aligned}
$$

Whence

$$
\begin{array}{ll}
\left(P_{x} M\right)<\left({ }_{x} M U\right), & x \leqq t \\
\left(P_{x} M\right)=\left({ }_{x} M U\right), & x=t+1 .
\end{array}
$$

Hence, the derivatives of order $t+1$ can be expressed as functions of the derivatives of lower order, and therefore the first of Lie's conditions is satisfied for $s=t+1$. 
9. The second of Lie's conditions for covariant equations. Considering the case that the first of Lie's conditions is satisfied, we start with (8.4), (8.9), (8.10) and the higher equations, deduced as explained in the preceding section:

$$
\begin{aligned}
& P \nabla_{\mu} v_{\lambda_{p}}=w_{\mu \lambda_{p}} ; \\
& P_{x} M \nabla_{\mu_{x}} v_{\lambda_{p}}=\nabla_{\mu_{x}} w_{\mu_{1} \lambda_{p}}+\stackrel{x}{T_{\mu_{x}} \lambda_{p}}{ }^{\nu_{p}} v_{\nu_{p}} \\
& +\stackrel{x}{T_{\mu_{x}} \dot{\lambda}_{p}}{ }^{\alpha_{1} \nu_{p}} \nabla_{\alpha_{1}} v_{\nu_{p}}+\cdots \\
& +\stackrel{x}{T_{\mu_{x}} \lambda_{p}}{ }^{\alpha_{x-1} \nu_{p}} \nabla_{\alpha_{x-1}} v_{\nu_{p}} \\
& (x=2, \cdots, q-1) \text {; } \\
& P_{q} M \nabla_{\mu_{q}} v_{\lambda_{p}}=\nabla_{\mu_{q}} w_{\mu_{1} \lambda_{p}}+\stackrel{q}{T_{\mu_{q}} \lambda_{p}} v_{\nu_{p}} \\
& +\stackrel{q}{T_{\mu_{q}} \dot{\lambda}_{p}}{ }^{\alpha_{1} \nu_{p}} \nabla_{\alpha_{1}} v_{\nu_{p}}+\cdots \\
& +\stackrel{q}{T}_{\mu_{q} \lambda_{p}}^{\alpha_{q-1} \nu_{p}} \nabla_{\alpha_{q-1}} v_{\nu_{p}} \text {. }
\end{aligned}
$$

The last equation, containing on the right side only derivatives up to the $(q-1)$ th, being solved with respect to $\nabla_{\left(\mu_{q}\right)} v_{h_{p}}$, we arrive at an equation of the form

$$
\nabla_{\mu_{q}} v_{\lambda_{p}}=Q_{\mu_{q} \lambda_{p}}
$$

$Q_{\mu_{q} \lambda_{p}}$ containing only derivatives up to the $(q-1)$ th. By differentiating (9.4) and alternating we have

$$
\frac{1}{2} R_{\omega \mu_{q} \lambda_{p}}^{\cdots \alpha_{q-1} \nu_{p}} \nabla_{\alpha_{q-1}} v_{\lambda_{p}}=\nabla_{[\omega} Q_{\left.\mu_{q}\right] \mu_{q-1} \lambda_{p}}
$$

The $q$ th derivatives on the right being eliminated using (9.4), this equation contains only derivatives up to the $(q-1)$ th. Hence it can be no algebraic consequence of (9.4). Differentiating (9.5) and eliminating always the $q$ th derivatives by means of (9.4) leads to an infinite series of equations containing derivatives only up to the $(q-1)$ th. Now either (9.1), (9.2), (9.5) and the equation obtained in this way are algebraically inconsistent, or they are algebraic consequences of a finite number of them. In the latter case we know by a general theorem proved by Delassus* that if one of the equations deduced from (9.5) by differentiation and elimination is an algebraic consequence of the preceding ones and (9.1), (9.2) and (9.5),

\footnotetext{
* Annales de l'Ecole Normale Supérieure, vol. 13 (1896), p. 449.
} 
then all following equations have the same property. Hence it is not necessary to consider these equations. In this latter case the second of Lie's conditions is satisfied because there is no other way of differentiating and eliminating of the sth derivatives than the way just shown.

Summing up, we have the following theorem:

$A$ system of covariant equations of the form

$$
\stackrel{\xi}{P} \nabla_{\mu} v_{\lambda_{p}}=\stackrel{\xi}{w}_{\mu \lambda_{p}}, \quad(\xi=1, \cdots, z)
$$

is equivalent to one equation of that form,

$$
P \nabla_{\mu} v_{\lambda_{p}}=w_{\mu \lambda_{p}}
$$

The equation being consistent and $U$ being an idempotent operator in $\lambda_{1} \ldots \lambda_{p}$ belonging to $P$, the general solution depends then and only then on a finite number of arbitrary constants, when there exists a number $q$ such that the characteristic numbers of the operators $P_{q} M$ and ${ }_{q} M U$ are equal:

$$
\left(P_{q} M\right)=\left({ }_{q} M U\right)
$$

If for every $x$

$$
\left(P_{x} M\right)<\left({ }_{x} M U\right)
$$

the general solution contains arbitrary functions of $x^{\nu}$.

10. The linear case. When $w_{\mu \lambda_{p}}$ is a linear function of $v_{\lambda_{p}}$ the equations (9.1) and (9.2) take the form*

$$
\begin{aligned}
& P \nabla_{\mu} v_{\lambda_{p}}=\stackrel{0}{u}_{\mu \lambda_{p}}+\stackrel{1}{u}_{\mu \lambda_{p}}^{\nu_{p}} v_{\nu_{p}}
\end{aligned}
$$

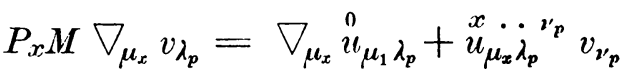

$$
\begin{aligned}
& +{\stackrel{x}{u_{\mu_{x}} \lambda_{p}} \boldsymbol{\alpha}_{1} \nu_{p}^{\prime}}^{u_{\alpha_{1}}} \nabla_{\nu_{p}}+\cdots
\end{aligned}
$$

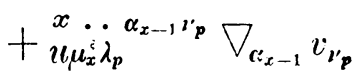

$$
\begin{aligned}
& (x=2, \cdots, q-1) \text {; }
\end{aligned}
$$

* The case in which the right side of (10.1) is zero, $p=2$, and $P$ is the operator mixing the suffixes $\mu \lambda_{1} \lambda_{2}$, is treated by Veblen and Thomas, these Transactions, vol. 25 (1923), pp. $599 \mathrm{ff}$. 


$$
\begin{aligned}
& P_{q} M \nabla_{\mu_{q}} v_{\lambda_{p}}=\nabla_{\mu_{q}}{\stackrel{0}{u_{\mu_{1}} \lambda_{p}}}+\stackrel{q}{u_{\mu_{q}} \lambda_{p}} \nu^{\nu_{p}} v_{\nu_{p}}
\end{aligned}
$$

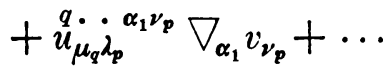

$$
\begin{aligned}
& +\stackrel{q q}{u_{\mu_{q}} \dot{\lambda}_{p}} \alpha_{q-1}^{\nu_{p}} \nabla_{\alpha_{q-1}} v_{\nu_{p}} ;
\end{aligned}
$$

where all quantities $u$ are functions of $x^{\nu}$ only, not containing $v_{\lambda_{p}}$ or derivatives of $v_{\lambda_{p}}$. The last equation contains only derivatives up to the $(q-1)$ th on the right. The solution of the last equation for ${ }_{q} M \nabla_{\mu_{q}} v_{\lambda_{p}}$ gives rise to an equation of the form

$$
\begin{aligned}
\nabla_{\mu_{q}} v_{\lambda_{p}}=\stackrel{0}{U}_{\mu_{q} \lambda_{p}} & +U_{\mu_{q} \lambda_{p}}^{\cdot \cdot{ }^{\nu_{p}}} v_{\nu_{p}} \\
& +U_{\mu_{q} \lambda_{p}}^{\cdot} \dot{\alpha}^{\alpha_{1} \nu_{p}} \nabla_{\alpha_{1}} v_{\nu_{p}}+\cdots \\
& +U_{\mu_{q} \lambda_{p}}^{\cdot} \dot{\alpha}^{\alpha_{q-1} \nu_{p}} \nabla_{\alpha_{q-1}} v_{\nu_{p}^{\prime}},
\end{aligned}
$$

containing only derivatives up to the $(q-1)$ th on the right. By differentiation and alternation of (10.4) and elimination of the $q$ th derivatives by means of (10.4) we get an equation of the form

$$
\begin{aligned}
& \stackrel{01}{V}_{\omega \mu_{q} \lambda_{p}}+\stackrel{1}{V}_{\omega \mu_{q} \lambda_{p}}^{\cdot \nu_{p}} v_{\nu_{p}}+\stackrel{1}{V}_{\omega \mu_{q} \dot{\lambda}_{p}}^{\alpha_{1} \nu_{p}} \nabla_{\alpha_{1}} v_{\nu_{p}}+\ldots \\
& +\stackrel{1}{V}_{\omega}^{*} \cdot \dot{\mu}_{q} \dot{\alpha}_{p}^{\alpha_{q-1} \nu_{p}} \nabla_{\alpha_{q-1}} v_{\nu_{p}}=0 .
\end{aligned}
$$

Differentiation of this equation and elimination of the $q$ th derivatives by means of (10.4) leads to an infinite set of equations of the same form

$$
\begin{aligned}
& \stackrel{0 y}{V}_{\omega_{y} \mu_{q} \lambda_{p}}+\stackrel{y}{V}_{\omega_{\omega_{y}} \mu_{q} \lambda_{p}}^{\nu_{p}} v_{\nu_{p}}+\stackrel{y}{V}_{\omega_{y} \mu_{q} \dot{\lambda}_{p}}^{\alpha_{1} \nu_{p} \nu_{p}} \nabla_{\alpha_{1}} v_{\nu_{p}}+\cdots \\
& +\stackrel{y}{V}_{\omega_{y} \mu_{q} \lambda_{p}}^{\alpha_{q-1} \nu_{p} p} \nabla_{\alpha_{q-1}} v_{\nu_{p}}=0 \quad(y=1,2, \cdots) .
\end{aligned}
$$

In all the preceding equations of this section quantities with a suffix 0 vanish for $\stackrel{0}{u}_{\mu \lambda_{p}}=0$. The quantities $U$ and $V$ in (10.4) and (10.6) are connected by the equations

$$
\begin{aligned}
& \stackrel{01}{V}_{\omega \mu_{q} \lambda_{p}}=\nabla_{[\omega} \stackrel{0}{U}_{\left.\mu_{q}\right] \mu_{q-1} \lambda_{p}}+\stackrel{0}{U}_{\left[\omega\left|\beta_{q-1} \gamma_{p}\right|\right.} U_{\left.\mu_{q}\right]}^{\cdot} \dot{\mu}_{q-1} \dot{\lambda}_{p}^{\beta_{q-1} \gamma_{p}},
\end{aligned}
$$

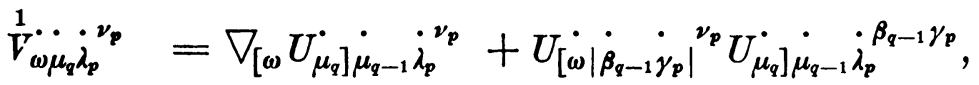

$$
\begin{aligned}
& \stackrel{1}{V}_{\omega \mu_{q} \lambda_{p}}^{\cdot \cdots} \alpha_{z} \nu_{p}=\nabla_{[\omega} U_{\left.\mu_{q}\right] \mu_{q-1}}^{\cdot} \dot{\lambda}_{p}^{\alpha_{z} \nu_{p}}+A_{[\omega}^{c_{s}} U_{\left.\mu_{q}\right] \mu_{q-1}}^{\cdot} \dot{\alpha}_{p}^{\alpha_{z-1} \nu_{p}} \\
& +U_{\left[\omega\left|\dot{\beta}_{q-1} \dot{\gamma}_{p}\right|\right.}^{\cdot \alpha_{s} \nu_{p}} U_{\left.\mu_{q}\right] \mu_{q-1}}^{\cdot} \dot{\lambda}_{p}^{\beta_{q-1} \gamma_{p}}-\frac{1}{2} \lambda R_{\omega \mu_{q} \lambda_{p}}^{\cdot{ }^{\alpha_{q-1} \nu_{p}}} \\
& (z=1, \cdots, q-1 ; \lambda=0, z<q-1 ; \lambda=1, z=q-1) \text {; }
\end{aligned}
$$




$$
\begin{aligned}
& \stackrel{0, y+1}{V_{\xi \xi\left(\omega_{y} \mu_{q} \lambda_{p}\right.}}=\nabla_{\xi} \stackrel{0 y}{\omega_{\omega_{y}} \mu_{q} \lambda_{p}}+\stackrel{0}{U_{\xi_{\beta_{q-1}} \gamma_{p}}} \stackrel{y}{V_{\omega_{y}} \dot{\mu}_{q} \dot{\lambda}_{p}} \boldsymbol{\beta}_{q-1} \gamma_{p},
\end{aligned}
$$

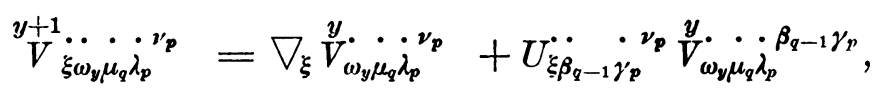

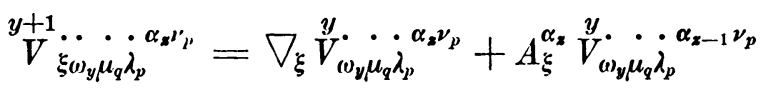

$$
\begin{aligned}
& +U_{\xi_{\beta_{q-1}} \dot{\gamma}_{p}}^{\cdot \alpha_{x} v_{p}} \stackrel{y}{V_{\omega_{y}}^{y} \dot{\mu}_{q} \lambda_{p}} \cdot \beta_{q-1} \gamma_{p} \\
& (y=1,2, \cdots ; \quad z=1, \cdots, q-1) \text {. }
\end{aligned}
$$

Now we regard the equations (10.1), (10.2) and the first $t$ equations (10.6) only from the algebraic point of view as equations for the determination of the unknown quantities $v_{\lambda_{p}}, \nabla_{\mu_{1}} v_{\lambda_{p}}, \cdots, \nabla_{\mu_{q-1}} v_{\lambda_{p}}$. These quantities must also satisfy the identities

$$
\begin{aligned}
\nabla_{\mu^{x}\left[\mu_{y} \mu_{x}\right] \mu_{x-1}} v_{\lambda_{p}} & =\frac{1}{2} \nabla_{\mu_{x}} R_{\mu_{y} \mu_{z} \lambda_{p}}^{\cdots \alpha_{z-1} \nu_{p}} \nabla_{\alpha_{z-1}} v_{\nu_{p}} \\
& =\frac{1}{2} \nabla_{\mu_{x}} R_{\mu_{y} \dot{\lambda}_{p}}^{\cdot{ }^{\alpha_{z-1}{ }^{\prime} p}} \nabla_{\boldsymbol{\alpha}_{z-1}} v_{\nu_{p}} ; \\
x & =2, \cdots, q-1 ; y=2, \cdots, x ; z=y-1) .
\end{aligned}
$$

We assume that (10.1), (10.2), the first $t$ equations (10.6) and (10.9) admit a solution $\stackrel{0}{v}_{\lambda_{p}}, \stackrel{0}{v}_{\mu_{1} \lambda_{p}}, \cdots, \hat{v}_{\mu_{q-1} \lambda_{p}}$. Then every solution of these equations can be written in the form

$$
v_{\mu_{y} \lambda_{p}}=\stackrel{0}{v}_{\mu_{y} \lambda_{p}}+\alpha v_{1}^{1} v_{\mu_{y} \lambda_{p}}+\cdots+\underset{s}{\alpha} v_{\mu_{y} \lambda_{p}}^{s} \quad(y=1, \cdots, q-1),
$$

where $\stackrel{1}{v}_{\mu_{y} \lambda_{p}}, \cdots, v_{\mu_{y} \lambda_{p}}, y=1, \cdots, q-1$ form a fundamental set of solutions of (10.9) and the reduced equations (10.1), (10.2), (10.6, first $t$ equations), viz. the equations found by setting $u_{\mu \lambda_{p}}=0$, and $\alpha, \cdots, \alpha$ are arbitrary functional parameters. Further we assume that the $(t+1)$ th equation $(10.6)$ is an algebraic consequence of (10.1), (10.2), (10.9) and the first $t$ equations (10.6). When the system (10.1), (10.2), (10.6), (10.9) is algebraically consistent, there will certainly be a number $t$ with these properties. Setting in the first $t$ equations (10.6) $v_{\lambda_{p}}=\stackrel{0}{v}_{\lambda_{p}}, \nabla_{\mu_{1}} v_{\lambda_{p}}=\stackrel{0}{v}_{\mu_{1} \lambda_{p}}$ etc., we have by differentiation

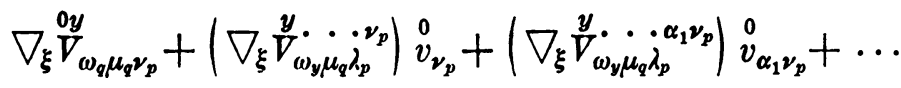

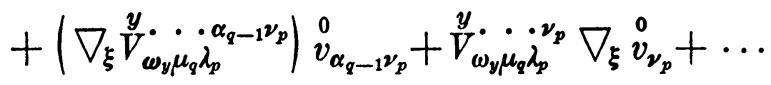

$$
\begin{aligned}
& +\stackrel{y}{V}_{\omega_{\nu} \mu_{q} \dot{\lambda}_{p}}^{\alpha_{q-1} \nu_{p}} \nabla_{\xi}{\stackrel{0}{v_{\alpha_{q-1}} \nu_{p}}}^{0}=0 \quad(y=1, \ldots, t) \text {. }
\end{aligned}
$$


Now the second to the $(t+1)$ th equation $(10.6)$ can be put into the form

$$
\begin{aligned}
& \nabla_{\xi} \stackrel{0 y}{V}_{\omega_{\omega_{y}} \mu_{q} \lambda_{p}}+\stackrel{0}{U}_{\xi_{\beta_{q-1}} \gamma_{p}} \stackrel{y}{V}_{\omega_{\omega_{y}} \mu_{q} \lambda_{p}}^{\beta_{q-1} \gamma_{p}}
\end{aligned}
$$

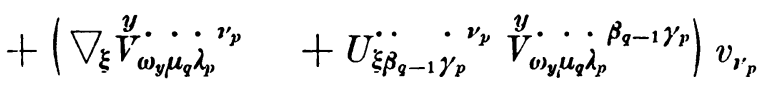

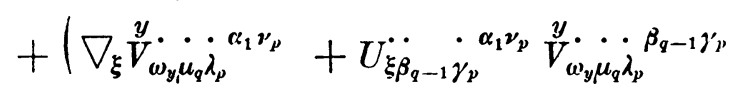

$$
\begin{aligned}
& \left.+A_{\xi}^{\alpha_{1}} \stackrel{y}{V_{\omega_{y}} \mu_{q} \lambda_{p}} \dot{\nu}_{p}\right) v_{\alpha_{1} \nu_{p}}+\cdots
\end{aligned}
$$

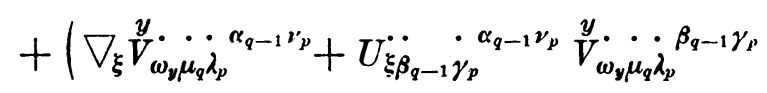

$$
\begin{aligned}
& \left.+A_{\xi}^{\alpha_{q-1}} \stackrel{y}{V}_{\omega_{y} \mu_{q} \lambda_{p}}^{\ldots} \alpha_{q-2} \nu_{p}\right) v_{\alpha_{q-1} \nu_{p}}=0, \\
& y=1, \ldots, t,
\end{aligned}
$$

in consequence of (10.8). Setting in (10.12)

$$
\begin{aligned}
v_{\lambda_{p}} & =\stackrel{0}{v}_{\lambda_{p}}, \\
\nabla_{\mu_{1}} v_{\lambda_{p}} & =\stackrel{0}{v}_{\mu_{1} \lambda_{p}} \text { etc. }
\end{aligned}
$$

and subtracting (10.11) from the so obtained equations we have

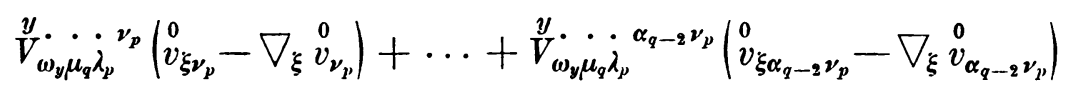

$$
\begin{aligned}
& +\left.\stackrel{y}{V} \cdot \underset{\omega_{y} \mu_{q} \lambda_{p}}{\cdots \alpha_{q-1} \nu_{p}}\right|_{U_{\xi \alpha_{q-1} \nu_{p}}^{0}} ^{0}+U_{\xi \alpha_{q-1} \nu_{p}}^{. \cdot \gamma_{p}}{\stackrel{\gamma}{\gamma_{p}}}_{v_{p}}^{0}+\cdots
\end{aligned}
$$

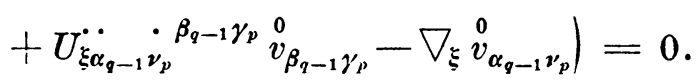

Hence the quantities

$$
\begin{aligned}
& a^{\xi}\left(v_{\xi \lambda_{p}}^{0}-\nabla_{\xi}{\stackrel{0}{v_{p}}}\right) \\
& a^{\xi}\left({\stackrel{0}{v_{\xi \alpha_{q}-2} \lambda_{p}}}^{0}-\nabla_{\xi}{\stackrel{0}{v_{\alpha_{q-2}} \lambda_{p}}}\right)
\end{aligned}
$$

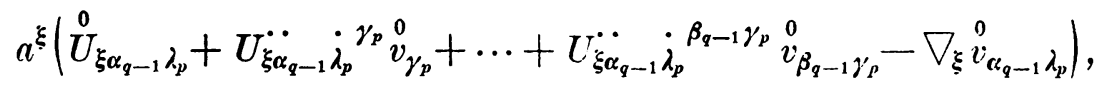

where $a^{\xi}$ is an arbitrary vector, form a solution of the first $t$ reduced equations (10.6). We will now prove that these same quantities also satisfy (10.9) and the reduced equations (10.1), (10.2).

Because of (10.9) the equations $(10.1,2,3)$ are equivalent to equations of the form

$$
\begin{aligned}
& P \nabla_{\mu_{x}} v_{\lambda_{p}}=\nabla_{\mu_{x}}{\stackrel{0}{\mu_{1} \lambda_{p}}}+\stackrel{x}{W_{\mu_{\alpha}}^{*} \lambda_{p}{ }^{\prime \prime}{ }^{\prime}} v_{\nu_{p}}+\cdots \\
& +\stackrel{x}{W}_{\mu_{x} \lambda_{p}}^{\cdot{ }^{\alpha_{x-1}} \nu_{p}} \nabla_{\alpha_{x-1}} v_{\nu_{p}}=0 \quad(x=1, \cdots, q),
\end{aligned}
$$


where

$$
\begin{aligned}
& \stackrel{x+1}{W_{\xi \mu_{x} \lambda_{p}}} \dot{\nu}_{p}=\nabla_{\xi} \stackrel{x}{W}_{\mu_{x} \lambda_{p}}^{\nu_{p}} \\
& \stackrel{x+1}{W_{\xi}^{\prime} \ddot{\mu}_{x} \lambda_{p}{ }^{\alpha} \nu_{p} \nu_{p}}=\nabla_{\xi} \stackrel{x}{W_{\mu_{x}}^{*} \dot{\lambda}_{p}^{\alpha_{y} \nu_{p}}}+A_{\xi}^{\alpha_{y}} \stackrel{x}{W}_{\mu_{x} \lambda_{p}}^{\alpha_{y-1} \nu_{p}},
\end{aligned}
$$

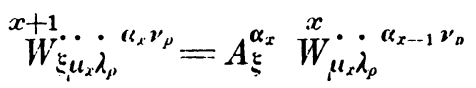

$$
\begin{aligned}
& (x=1, \cdots, q-1 ; y=1, \cdots, x-1) \text {. }
\end{aligned}
$$

Substituting $\stackrel{0}{v}_{\lambda_{p}}, \stackrel{0}{v}_{\mu_{1} \lambda_{p}}$ etc. in the first $q-1$ equations $(10.15)$ we have by differentiation of the first $q-2$ equations

$$
\begin{aligned}
& P \nabla_{\xi}{\stackrel{0}{v_{\mu} \lambda_{p}}}=\nabla_{\xi \mu_{x}}{\stackrel{0}{u_{\mu_{1} \lambda_{p}}}}+\left(\nabla_{\xi} \stackrel{x}{W}_{\mu_{\mu_{x}} \dot{\lambda}_{p}}^{\nu_{p}}\right){\stackrel{0}{v_{\nu_{p}}}}+\stackrel{x}{W}_{\mu_{x} \dot{\lambda}_{p}}^{\nu_{p}} \nabla_{\xi}{\stackrel{0}{v_{\nu_{p}}}}+\cdots
\end{aligned}
$$

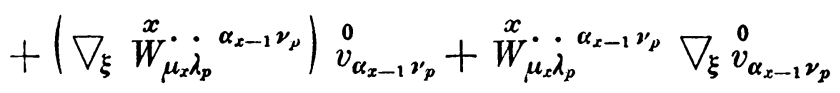

$$
\begin{aligned}
& (x=1, \cdots, q-2) \text {. }
\end{aligned}
$$

Subtracting these equations from the second to the $(q-1)$ th equation

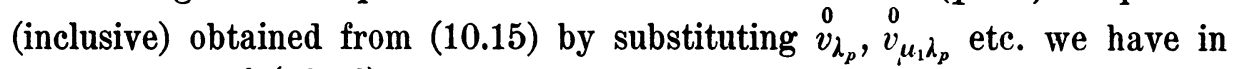
consequence of (10.16)

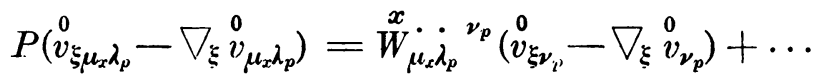

$$
\begin{aligned}
& +\stackrel{x}{W}_{\mu_{x} \dot{\lambda}_{p}}^{\alpha_{x-1} \nu_{p}}\left({\stackrel{0}{v} \boldsymbol{\xi}_{x-1} \nu_{p}}^{0}-\nabla_{\xi}{\stackrel{0}{\alpha_{x-1} \nu_{p}}}^{0}\right) \quad(x=1, \cdots, q-2) .
\end{aligned}
$$

Differentiating the $(q-1)$ th equation (10.15) after having substituted $\stackrel{0}{v}_{\lambda_{p}}$ etc. in it, we have

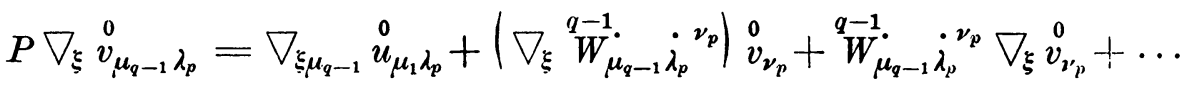

$$
\begin{aligned}
& +\left(\nabla_{\xi} \stackrel{q-1}{W}_{\mu_{q-1}}^{\cdot} \dot{\lambda}_{p}^{\alpha_{q-2} \nu_{p}}\right){\stackrel{0}{\alpha_{\alpha-2} \nu_{p}}}+\stackrel{q-1}{W}_{\boldsymbol{\mu}_{q-1} \dot{\lambda}_{p}}^{\alpha_{q-2} \nu_{p}} \nabla_{\xi}{\stackrel{0}{v_{\alpha_{q-2} \nu_{p}}}}
\end{aligned}
$$

or, in consequence of (10.16),

$$
\begin{aligned}
& P \nabla_{\xi}{\stackrel{0}{v_{\mu_{q-1}} \lambda_{l^{\prime}}}}-\nabla_{\xi_{\mu_{q-1}}}{\stackrel{0}{u_{\mu_{1}} \lambda_{p}}}-\stackrel{q}{W}_{\boldsymbol{\xi}_{\mu_{q-1}}}^{.} \dot{\lambda}_{p}^{\nu_{p}}{\stackrel{0}{v_{\nu_{p}}}}
\end{aligned}
$$

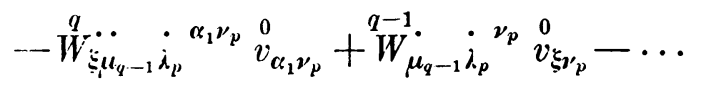

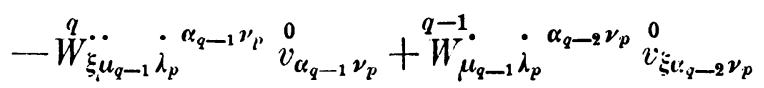

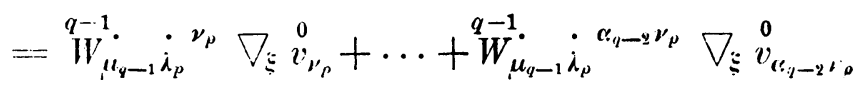


Now the $q$ th equation (10.15) is equivalent to (10.4). Hence

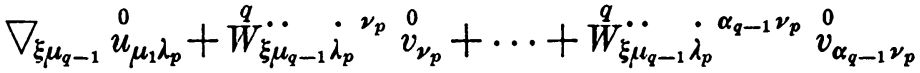

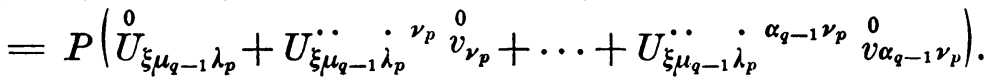

Hence (10.19) is equivalent to

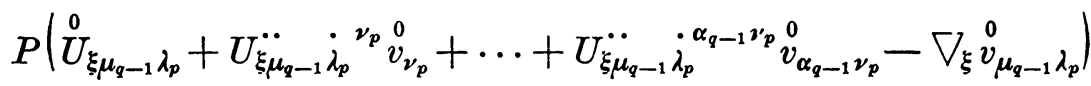

$$
\begin{aligned}
& =\stackrel{q-1}{W_{\mu_{q-1}}^{\cdot}} \dot{\lambda}_{p}^{\nu_{p}}\left({ }_{v_{\xi \nu_{p}}}^{0}-\nabla_{\xi}{\stackrel{0}{\nu_{\nu}}}_{\nu_{p}}\right)+\cdots+\stackrel{q-1}{W_{\mu_{q-1}}^{\cdot}} \dot{\lambda}_{p}^{\alpha_{q-2} \nu_{p}}\left({ }_{v_{\xi \alpha_{q-2} \nu_{p}}^{0}}^{0}-\nabla_{\xi}{\stackrel{0}{\alpha_{q-2} \nu_{p}}}^{0}\right) .
\end{aligned}
$$

From (10.18) and (10.22) it follows that the quantities (10.14) satisfy the reduced equations $(10.1,2)$, q. e. d.

Substituting $v_{\lambda_{p}}$ etc. in (10.9) we obtain

$$
\begin{aligned}
& {\stackrel{0}{v_{\mu_{x}}\left[\mu_{y} \mu_{z}\right] \mu_{x-1} \lambda_{p}}}=\frac{1}{2} \nabla_{\mu_{x}}^{\prime} R_{\mu_{y} \lambda_{p}}^{\cdot \alpha_{z-1} \nu_{p}}{\stackrel{0}{v_{\alpha_{z-1}} \nu_{p}}}^{0} \\
& (x=2, \cdots, q-1 ; \quad y=2, \cdots, x ; \quad z=y-1) \text {, }
\end{aligned}
$$

where on the right side $\nabla^{\prime}$ is a real differential operator equivalent to $\nabla$

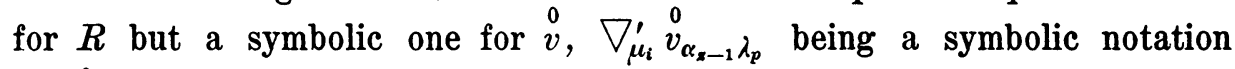
for ${\stackrel{0}{v_{\mu_{i} \alpha_{x-1}} \lambda_{p}}}_{i}, i=0, \ldots, x$. Differentiating the first $q-3$ equations (10.23) for some fixed values of $y$ we have

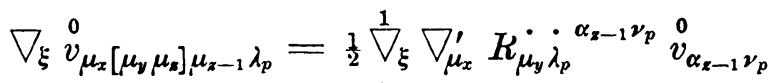

$$
\begin{aligned}
& +\frac{1}{2} \stackrel{2}{\nabla}_{\xi} \nabla_{\mu_{x}}^{\prime} R_{\mu_{y} \lambda_{p}}^{\alpha_{z-1} \nu_{p}}{\stackrel{0}{v_{\alpha_{z-1} \nu_{p}}}} \\
& (x=2, \cdots, q-2 ; y=2, \cdots, x ; z=y-1)
\end{aligned}
$$

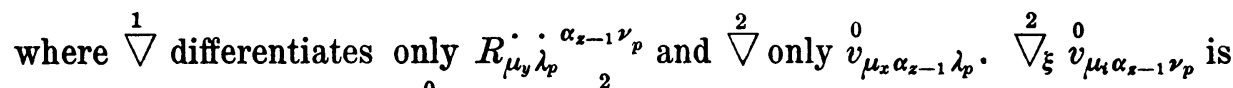

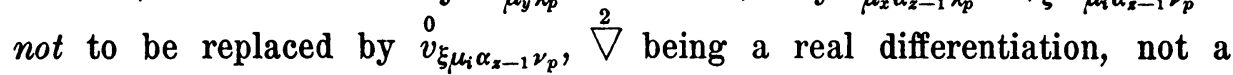
symbolic one. Subtracting these equations from the second to the $(q-1)$ th equation (10.23) (inclusive) for the same value of $y$ we obtain

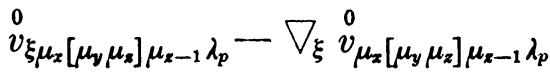

$$
\begin{aligned}
& =\frac{1}{2} \nabla_{\xi}^{\prime} \nabla_{\mu_{x}}^{\prime} R_{\mu_{y} \lambda_{p}}^{\cdot \alpha_{x-1} \nu_{p}}{\stackrel{0}{v_{\alpha_{z-1}} \nu_{p}}}^{0} \\
& -\frac{1}{2} \nabla_{\xi} \nabla_{\mu_{x}}^{\prime} R_{\mu_{y} \lambda_{p}}^{\alpha_{z-1} \nu_{p}}{\stackrel{0}{v_{\alpha_{z-1}} \nu_{p}}} \\
& -\frac{1}{2} \stackrel{2}{\nabla}_{\xi} \nabla_{\mu_{x}}^{\prime} R_{\mu_{y} \dot{\lambda}_{p}}^{\alpha_{z-1} \nu_{p}}{\stackrel{0}{v_{\alpha_{z-1} \nu_{\rho}}}}^{0} \\
& =\frac{1}{2}\left(\stackrel{\nabla}{\nabla}_{\xi}^{\prime}-\stackrel{2}{\nabla}_{\xi}\right) \nabla_{\mu_{x}}^{\prime} R_{\mu_{y} \lambda_{p}}^{\alpha_{x-1} \nu_{p}}{\stackrel{0}{v_{\alpha_{k-1}} \nu_{p}}} .
\end{aligned}
$$


Differentiating the $(q-2)$ th equation (10.23) for some fixed value of $y$ we have

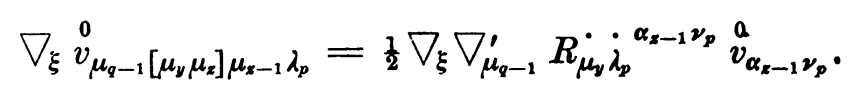

Now from (10.4) we deduce

hence it follows that

$$
\begin{aligned}
& \nabla_{\xi \mu_{q-1}\left[\mu_{y} \mu_{z}\right] \mu_{z-1}} v_{\lambda_{p}}=\frac{1}{2} \nabla_{\xi \mu_{q-1}} R_{\mu_{\nu} \dot{\lambda}_{p}}^{\alpha_{z-1} \nu_{p}} \nabla_{\alpha_{z-1}} v_{\nu_{p}}
\end{aligned}
$$

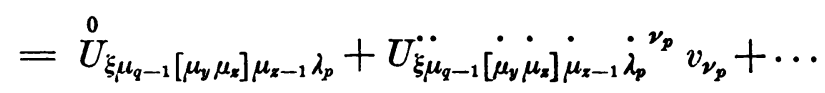

$$
\begin{aligned}
& +U_{\xi \mu_{q-1}\left[\mu_{\nu} \dot{\mu_{z}}\right] \dot{\mu}_{z-1}}^{\ddot{\lambda_{p}}} \dot{\alpha}_{p}^{\alpha_{q-1} \nu_{p}} \nabla_{\alpha_{q-1}} v_{\nu_{p}},
\end{aligned}
$$

$$
\begin{aligned}
& \stackrel{0}{U}_{U_{\mu_{q-1}}\left[\mu_{y} \mu_{z}\right] \mu_{z-1} \lambda_{p}}=0, \\
& U_{\xi \mu_{q-1}\left[\mu_{y} \mu_{z}\right] \dot{\mu}_{z-1} \dot{\lambda}_{p}}^{\ddot{\alpha_{i} \nu_{p}}}=0 \quad(i=1, \ldots, z-2, q-1),
\end{aligned}
$$

and that there exist such relations between the quantities $U$ and $R$ in (10.27) that for every set of quantities ${\stackrel{0}{v_{p}}}_{\text {, }}$, etc. which satisfies (10.9) the following equation holds:

$$
\begin{aligned}
& \frac{1}{2} \nabla_{\xi_{\mu_{q-1}}}^{\prime} R_{\mu_{y} \dot{\lambda}_{p}}^{\alpha_{z-1} \nu_{p}}{\stackrel{0}{v_{\alpha_{z-1}} \nu_{p}}}
\end{aligned}
$$

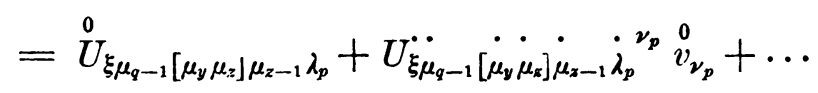

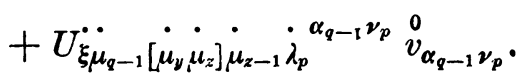

From (10.26) and (10.29) we get

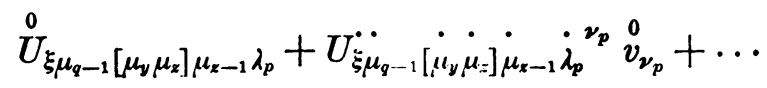

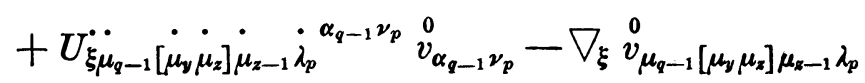

$$
\begin{aligned}
& =\frac{1}{2}\left(\stackrel{2}{\nabla}-\stackrel{2}{\nabla} \nabla_{\xi}\right) \nabla_{\mu_{q-1}}^{\prime} R_{\mu_{y} \lambda_{p}}^{\cdot \alpha_{z-1} \nu_{p}}{\stackrel{0}{v_{\alpha_{z-1}} \nu_{p}}}^{0}
\end{aligned}
$$

From (10.25), (10.27) and (10.30) follows that the quantities (10.14) satisfy also the equations (10.9), q.e.d. Hence these quantities satisfy the equations (10.9) and the reduced equations $(10.1,2)$ and (10.6, first $t$ equations). The same can be proved in like manner for the quantities 
$(10.31)$

$$
\begin{aligned}
& a^{\xi}\left({\stackrel{a}{\xi} \alpha_{q-2} \lambda_{p}}_{p}^{a}-\nabla_{\xi}{\stackrel{a}{v_{\alpha_{q-2} \lambda_{p}}}}^{a}\right),
\end{aligned}
$$

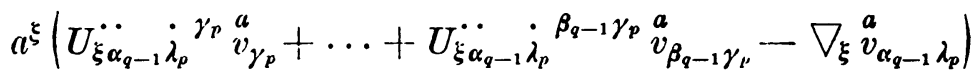

$$
\begin{aligned}
& (a=1, \cdots, s) \text {. }
\end{aligned}
$$

A fundamental set of solutions of the reduced equations $(10.1,2)$ and (10.6, first $t$ equations) being $v_{\lambda_{p}}, \cdots, v_{\lambda_{p}}$, etc., there must exist equations of the form

$$
\begin{aligned}
& i_{\xi \nu_{p}}^{i}-\nabla_{\xi} i_{\nu_{p}}^{i}=\sum_{b}^{1, \cdots, s}{ }^{i b} p_{\xi}^{b} v_{\nu_{\rho}}
\end{aligned}
$$

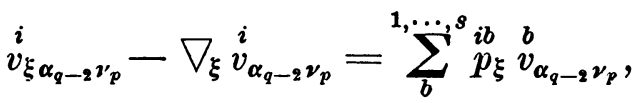

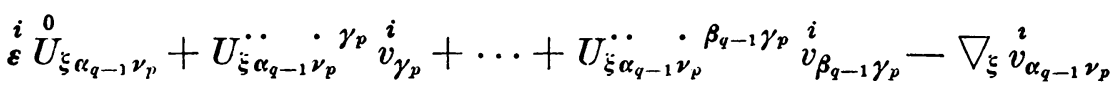

$$
\begin{aligned}
& =\sum_{l}^{1, \cdots i} p_{\xi}^{s} v_{\alpha_{q-1} \nu_{p}} \\
& \left(i=0,1, \ldots, s ; \quad \stackrel{i}{\varepsilon}=\left\{\begin{array}{l}
1, i=0 \\
0, i \neq 0
\end{array}\right) .\right.
\end{aligned}
$$

By differentiation and alternation we get from (10.32)

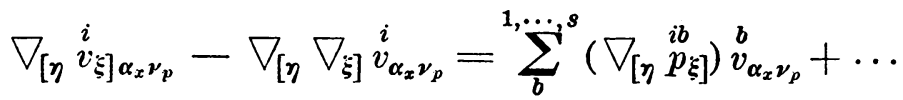

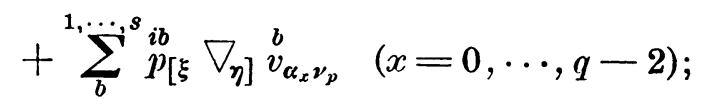

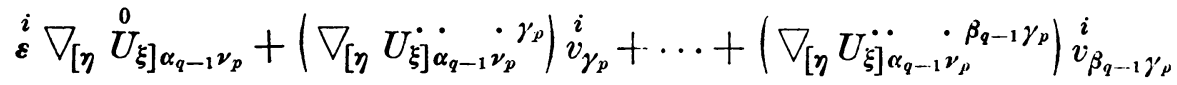

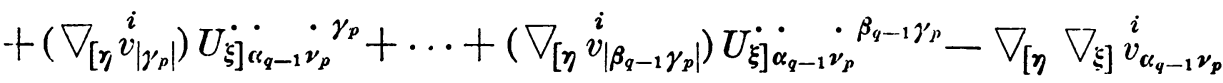

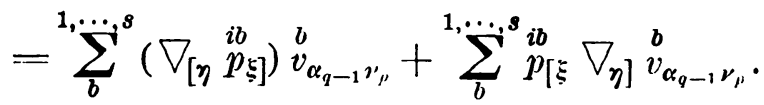

Because of (10.32), (10.7) and (10.5), we get from (10.33)

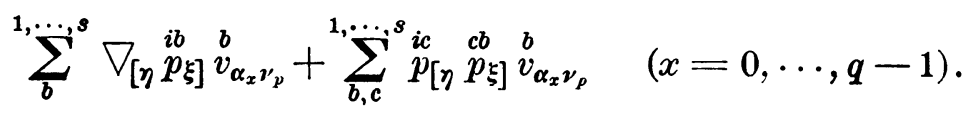


Hence

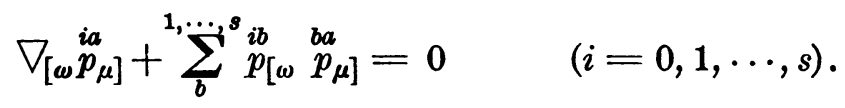

Now we will prove that the general solution of (10.1) has the form

$$
v_{\lambda_{p}}=\stackrel{0}{v}_{\lambda_{p}}+\underset{1}{\alpha} v_{\lambda_{p}}^{1}+\cdots+\underset{s}{\alpha} v_{\lambda_{p}}^{s}
$$

and that the parameters $\alpha$ can be found by the integration of a completely integrable system. In order that $(10.1)$ be satisfied by (10.36) it is necessary and sufficient that

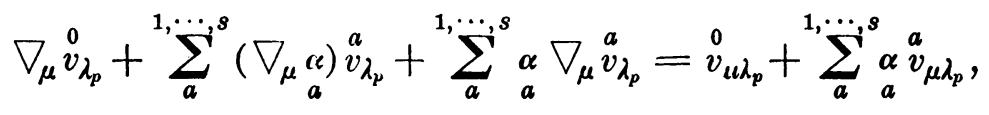

or, because of (10.32),

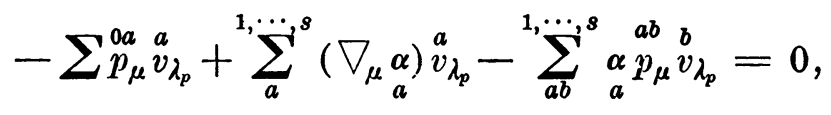

or

$$
\nabla_{\lambda} \underset{a}{\alpha}=\stackrel{0 a}{p}_{\lambda}+\sum_{b}^{1, \cdots_{b}^{s}} \underset{b}{a b} p_{\lambda}
$$

These equations having the same form as (3.13) are completely integrable.

Hence the integration of (10.1) is reduced to the algebraic solution of the system $(10.1,2,6,9)$ and the integration of the completely integrable system (10.38).

Delft, Holland. 